\title{
Retention of Nanoparticles: From Laboratory Cores to Outcrop Scale
}

\author{
Harpreet Singh and Farzam Javadpour \\ Bureau of Economic Geology, Jackson School of Geosciences, University of Texas at Austin, Austin, TX, USA \\ Correspondence should be addressed to Farzam Javadpour; farzam.javadpour@beg.utexas.edu
}

Received 11 April 2017; Accepted 8 May 2017; Published 10 July 2017

Academic Editor: Jianchao Cai

Copyright (C) 2017 Harpreet Singh and Farzam Javadpour. This is an open access article distributed under the Creative Commons Attribution License, which permits unrestricted use, distribution, and reproduction in any medium, provided the original work is properly cited.

\begin{abstract}
Laboratory experiments on small scale core plugs have shown controlled nanoparticles (NPs) retention. The length scale of subsurface media where NPs must be transported is an important factor that should be accounted for in a comprehensive manner when translating laboratory results to field scale. This study investigates the fraction of NPs retained inside porous media as a function of length scale of the media. A two-dimensional numerical model was used to simulate the retention of NPs at multiple scales of porous media, starting from laboratory scale cores to heterogeneous outcrop scales. Retention of NPs is modeled based on the concept of reversible and irreversible retention, by using the laboratory scale determined parameters. Our results show that the fraction of retained NPs increases nonlinearly with the length scale of the homogeneous media. The results also show that if the heterogeneity of the medium is consistent across scales, the fraction of retained NPs would behave just like homogeneous medium. In this study, small change in heterogeneity at two outcrop scales affects the retention of NPs, suggesting that heterogeneity may significantly impact the retention behavior of NPs that may not necessarily follow the behavior predicted from homogeneous cores (or periodically heterogeneous medium).
\end{abstract}

\section{Introduction}

Engineered nanoparticles (NPs) carry unique properties that make them quite attractive for applications in different industries. Even though they have been widely used in various industries such as the medical field for enhanced imaging $[1,2]$, targeted drug-delivery, and biosensing [3-5], NPs have yet to be adapted for their potentially attractive application in porous media. These applications may include enhanced hydrocarbon recovery through nanosuspensions [6-9], subsurface mapping through nanosensors $[10,11]$, hydraulic fracture characterization through nanomagnetization of reservoirs [12], hydraulic fracturing fluid with better proppant transport ability through nanofoams [13], and convective heat transfer [14, 15]. Many of these applications within porous media, such as enhanced oil recovery and subsurface mapping, would require the NPs to travel the depth and breadth of the targeted reservoir zone, which typically extend to hundreds of feet. Apart from economics, their use has been mainly constrained by technical challenges such as NP retention on the rock surface [16-19], which keeps it from propagating into the targeted zone. Other issues are specific to some reservoirs [20], but all these problems involve understanding the transport behavior of NPs inside porous media, the most important being the retention of NPs on the rock surface.

NPs have an affinity to retain inside porous media because of the gravitational force and chemical force exerted by the rock surface. The retention of NPs is a key concern for its potential applications in subsurface reservoir mapping, characterization, and enhanced hydrocarbon recovery. Surface properties of the NPs can significantly affect retention process at the laboratory scale, for example, up to $3 \%$ retention for silica NPs $[19,21,22], 0.3 \%$ retention for polymeric nanospheres [23], and up to $60 \%$ retention for nano- $\mathrm{TiO}_{2}[13,23]$.

Many laboratory experiments have been performed to characterize retention of NPs in a porous medium [24-31], and retention of NPs has been found to be controlled by the grain size [13], flow direction due to gravity settling [32], and roughness of the rock matrix, in addition to the chemical 
properties of the NPs [27, 31]. These results have led to further related studies that focus on the effect of various sedimentary rocks $[17,21,22]$ on the retention of NPs. Past studies have used many different models to study the retention of NPs on the rock surface. Although many laboratory experiments have been conducted and some analytical models [33-38] have been proposed to investigate the retention of NPs, very few numerical models have been developed to simulate their retention. These numerical models include those by Li et al. [36], Cullen et al. [39], Zhang [19], El-Amin et al. [40], and Becker et al. [41]. However, these numerical models were developed mostly to validate one-dimensional laboratory data using homogeneous media, and there has been no study where such laboratory tested model was applied to a field scale scenario.

Some limitations of the above numerical modeling studies are that they have been applied (1) only to laboratory scale data, (2) in porous media having homogeneous physical and chemical characteristics, and (3) in studies of only onedimensional media. Laboratory experiments are helpful in understanding the physics and chemistry of NP transport and retention; however, the experimental conditions used, specifically the flow velocity, are not representative of subsurface field conditions in many cases. Because conducting pilot tests to study NP transport is expensive and time consuming, numerical models validated by laboratory scale data can be used to model typical field scale scenarios with appropriate parameters. There are very few studies that model the mobility of NPs at field scale, and specifically the effect of NPs retention at field scale is even less studied. One important study in this area is by Cullen et al. [39], which models the mobility of carbon NPs and nanofullerenes at a pilot scale $(8 \mathrm{~m} \times 10 \mathrm{~m})$ using a two-dimensional numerical model, although numerical models have been used to investigate colloid transport on a slightly bigger scale than the laboratory [42] by employing two- and three-dimensional models with a limited representation of physical and chemical heterogeneity. Singh et al. [43] also performed field scale simulations using uranium NPs for enhanced storage of $\mathrm{CO}_{2}$ in geologic formations, but their study did not consider the retention of NPs. Although there are many laboratory scale studies for NPs retention, there are not many studies that investigate NPs retention at field scale. To our knowledge, study by Cullen et al. [39] is one well-known study that specifically models $\mathrm{NP}$ retention at a size $(8 \mathrm{~m} \times 10 \mathrm{~m})$ larger than laboratory scale. Even though they complement their numerical model with observations from pilot-scale studies, their numerical modeling has limited representation of a real field scenario. For example, Cullen et al. [39] do not account for the heterogeneity of the chemical attributes of the rock, which primarily control the retention of NPs under field conditions. Cullen et al. [39] use a very basic heterogeneity structure of hydraulic conductivity, and they do not explain the structure of the heterogeneity, which does not seem to be from any real field scenario. Also, Cullen et al. [39] used a colloid filtration model with a site-blocking term to simulate NP retention, but since their study new retention models have been proposed that capture the transport of NPs better than the colloid filtration model $[19,41,44-46]$.
To overcome the limitations discussed above and to capture the effect of NP retention at field scales, an important step toward its field implementation, further work is necessary to explore these issues of length scales representative of real field conditions. Although, many experimental studies have furthered our understanding of the mechanisms governing the retention of NPs in the porous media, studying the retention at field scales with heterogeneous porous media is only possible through numerical investigations. The objective of this work is to explore NP retention at increasing length scales from laboratory to field, while honoring the physical and chemical heterogeneity corresponding to those scales.

\section{Theory and Modeling}

2.1. Transport Equation. We consider the transport of NPs by an aqueous fluid through heterogeneous, two-dimensional, saturated porous media by mechanisms of advection, dispersion, and retention (ADR). We consider this transport on a continuum scale, where the fluid and reservoir parameters are regarded as averages over a representative elementary volume. The governing equation for transport of NPs in porous media is obtained by the conservation of the concentration of fluid species (NPs) and is given as follows:

$$
\begin{aligned}
\text { Mass Balance: } \frac{\partial c}{\partial t}+\nabla \cdot(v c-D \nabla c) & =\left(\frac{\rho_{b}}{\phi}\right) \frac{\partial s}{\partial t} \Longrightarrow \\
\frac{\partial c}{\partial t}+\operatorname{div} \cdot(v c)-\operatorname{div} \cdot(D \nabla c) & =\left(\frac{\rho_{b}}{\phi}\right)\left(\frac{\partial s}{\partial t}\right),
\end{aligned}
$$

where $c\left[\mathrm{M} / \mathrm{L}^{3}\right]$ is the concentration of NPs in the fluid, $t$ [T] is time, div is divergence, $v[\mathrm{~L} / \mathrm{T}]$ is the fluid velocity inside the porous medium (interstitial velocity), $D\left[\mathrm{~L}^{2} / \mathrm{T}\right]$ is the hydrodynamic dispersion coefficient, $s[\mathrm{M} / \mathrm{M}]$ is the concentration of the retained NPs, $\rho_{b}\left[\mathrm{M} / \mathrm{L}^{3}\right]$ is the bulk density of the porous media (system of matrix and fluid), and $\phi$ is the porosity of the medium. The conservation term on the right-hand side of the equation assumes that retention of NPs to the rock is free of any chemical reactions between the fluid and the rock.

The three terms on the left-hand side of (2) represent the rate of change of concentration, the component of concentration transported by advection, and the component of concentration transported by diffusion, respectively. The term on the right-hand side of (2) represents the component of concentration released (or retained) owing to retention of NPs in porous media.

On the basis of the NP retention mechanism, there can be different expressions for the source term in the transport equation. A comprehensive and detailed study by Zhang [19] compares five different types of one-dimensional retention models: colloid filtration model, Langmuir sorption model (generally used in gas adsorption/desorption, e.g., [47, 48]), two-step model, two-rate model, and two-site model, in which the simulated effluent history from each retention model is matched against the laboratory scale experimental data. Zhang concluded that, among the five retention models, the two-site model has the capability to capture the most 


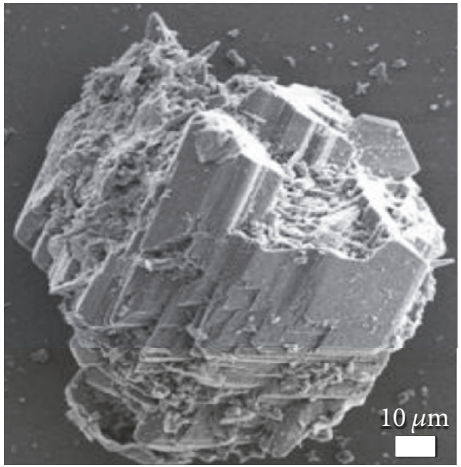

(a)

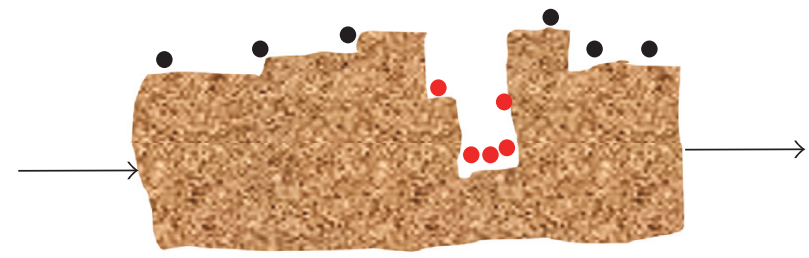

(b)

FIGURE 1: Irreversible and reversible retention sites for NPs illustrated using (a) a SEM picture of a sand grain from crushed Boise sandstone sieved between the size range of 90-105 $\mathrm{m}$ (adapted from [46]) and (b) a schematic of rough sand grain surface with NPs. Red circles represent NPs trapped permanently, whereas black circles represent NPs that have the potential to escape from their site of retention.

features of NP transport in porous media. A truncated form of the two-site model was used by Becker et al. [41] to model the retention of NPs and stabilizing agents in porous media. Therefore, based on the above understanding of NPs retention, we chose the two-site model to model the retention behavior of NPs in porous media.

\subsection{Retention Model for Mass Balance. Retention of many} NPs, including carbon NPs within porous media, depicts a hysteresis behavior, as observed experimentally by several authors [24-26, 28, 30], suggesting that retention of NPs is not an entirely irreversible process. For instance, NP effluent history on postflush typically depicts a tail having a larger concentration when compared with the tracer [19], suggesting that some of the retained NPs that get desorbed on postflush were part of the reversible retention. Therefore, on the basis of NP retention research from the past few years, it is now commonly accepted in most of the recent studies [44] that, out of the total NPs retained inside a porous medium, some portion would desorb through a reversible process while the remaining NPs would stay retained irreversibly. This behavior of NPs has been shown to be captured by the two-site retention model [46] and has also been shown to work better than the other retention models that simulate NP retention. Some of those models were also reviewed by Goldberg et al. [49], whose findings confirm with the study of Zhang et al. [46]. The other retention models that have been used in the past include (i) the colloid filtration model, (ii) filtration model with site blocking, (iii) filtration model with detachment, and (iv) kinetic Langmuir model.

The two-site model is an extension of the modified versions of the colloid filtration model used by Cullen et al. [39] who introduced a maximum retention capacity with a site-blocking term and by Wang et al. [50] who introduced a reversible retention term to simulate fullerene NPs transport. These modified colloid filtration models have been used with success to simulate multiwalled carbon nanotube (MWCNT) through columns packed with glass beads or sandstone [51]. The physical interpretation of the two-site model comes from the rough grain surfaces that include corners, crevices, and fissures (as shown by Figure 1(a)) that may retain the NPs and act as impediments to their flow. The two-site retention model assumes that there are two types of sites inside a watersaturated porous media that control the overall retention of NPs. These sites are functions of physical heterogeneity and roughness of the porous medium, in addition to chemical heterogeneity of the retention sites. These two sites control the retention of NPs through two mechanisms:

(i) Irreversible Retention. This retention mechanism occurs in regions having some cavity in which NPs get trapped permanently and cannot escape under any realistic fluid flow conditions.

(ii) Reversible Retention. This retention mechanism occurs in regions having relatively flat and rough surfaces that could allow NPs to detach and escape under realistic fluid flow conditions.

The above two mechanisms are illustrated schematically in Figure 1(b), which shows the NPs trapped by two types of sites that control the overall retention of NPs.

The two sites controlling the retention are independent of each other; therefore, the governing equation of the two-site model is described as follows:

$$
\left(\frac{\rho_{b}}{\phi}\right)\left(\frac{\partial s_{t}}{\partial t}\right)=\left(\frac{\rho_{b}}{\phi}\right)\left(\frac{\partial s_{1}}{\partial t}\right)+\left(\frac{\rho_{b}}{\phi}\right)\left(\frac{\partial s_{2}}{\partial t}\right),
$$

Irreversible retention: $\left(\frac{\rho_{b}}{\phi}\right)\left(\frac{\partial s_{1}}{\partial t}\right)$

$$
=k_{\mathrm{irr}}\left(1-\frac{s_{1}}{s_{1 \max }}\right) c
$$

$$
\begin{aligned}
& \text { Reversible retention: }\left(\frac{\rho_{b}}{\phi}\right)\left(\frac{\partial s_{2}}{\partial t}\right) \\
& =k_{\mathrm{ra}}\left(1-\frac{s_{2}}{s_{2 \max }}\right) c-\left(\frac{\rho_{b}}{\phi}\right) k_{\mathrm{rd}} s_{2},
\end{aligned}
$$


where $k_{\mathrm{irr}}[1 / \mathrm{T}]$ is the irreversible retention rate constant, $k_{\mathrm{ra}}$ $[1 / \mathrm{T}]$ is the reversible retention rate constant, $k_{\mathrm{rd}}[1 / \mathrm{T}]$ is the desorption rate constant, $s_{1 \max }[\mathrm{M} / \mathrm{M}]$ and $s_{2 \max }[\mathrm{M} / \mathrm{M}]$ are the capacities for irreversible and reversible retention, respectively, and $s_{1}[\mathrm{M} / \mathrm{M}]$ and $s_{2}[\mathrm{M} / \mathrm{M}]$ are the concentration of retained NPs on the porous media matrix due to irreversible and reversible processes, respectively. If the retention capacities greatly exceed the concentration of the retained NPs, then (4) and (5) would reduce to first-order kinetics and in that case the rate of retention would vary linearly with the concentration of NPs in the fluid.

2.3. Numerical Solution Method. We discretize the above system of three coupled partial differential equations, (2), (4), and (5), using fully implicit finite-difference method with central difference in space and forward difference in time, including an upwinding scheme to account for the spatial heterogeneity of the porous medium.

2.3.1. Initial and Boundary Conditions. The initial conditions for the coupled system of three partial differential equations, (2), (4), and (5), are

$$
\begin{aligned}
& c(x, y, t=0)=0, \\
& s(x, y, t=0)=0 .
\end{aligned}
$$

Boundary conditions for the above ADS equation are

$$
\begin{array}{r}
c\left(x=1, y \in\left[1, L_{y}\right], 0<t<t_{\mathrm{inj}}\right)=c_{\mathrm{inj}}, \\
c\left(x=1, y \in\left[1, L_{y}\right], t>t_{\mathrm{inj}}\right)=0, \\
\frac{\partial c\left(x=L_{x}, y \in\left[1, L_{y}\right], t\right)}{\partial x}=0, \\
c\left(x \in\left[1, L_{x}\right], y=0, t\right)=0, \\
c\left(x \in\left[1, L_{x}\right], y=L_{y}, t\right)=0 .
\end{array}
$$

Here, $L_{x}[\mathrm{~L}]$ and $L_{y}[\mathrm{~L}]$ are length of the medium along $x$ and $y$ directions, respectively, $c_{\mathrm{inj}}\left[\mathrm{M} / \mathrm{L}^{3}\right]$ is the concentration of the NPs, and $t_{\text {inj }}[-]$ is the dimensionless time for which NPs are injected in terms of pore volumes (PVs).

2.4. Model Parameters. To accomplish the objective of this work, we need to establish a realistic range of model parameters that typically exist in the subsurface. In this regard, below we discuss in detail the procedure to determine each parameter required in the modeling and later summarize the data at core and field scales in two separate tables.

2.4.1. Fluid Velocity. The range of some parameters such as fluid velocity and diffusion constant at subsurface operating conditions is well known through years of research in hydrocarbon production and groundwater flow. The fluid velocity in the subsurface is much lower in magnitude $(\sim 0.01-1 \mathrm{ft} / \mathrm{d})$ than the fluid velocity used in most laboratory experiments $(>0.01 \mathrm{ft} / \mathrm{d})$.
2.4.2. Hydrodynamic Dispersion Coefficient. It has been proved that the effective hydrodynamic dispersion coefficient in the field is comparatively larger than the hydrodynamic dispersion coefficient in the laboratory [52-58]. This finding is based on a literature survey of 40 field tracer tests at 15 geologic sites. These tests were used to analyze the dispersion coefficient by two methods: (1) using directly reported data and (2) reanalyzing the corresponding field tracer tests using analytical and semianalytical methods. It was concluded that the larger value of the dispersion coefficient at field scale compared with that at laboratory scale can be attributed to many mass-transfer processes at different scales in naturally heterogeneous rock systems. The factor by which the dispersion coefficient in the field is greater than in the laboratory ranges from 1 to 10,000 for observation scales from 5 to $2000 \mathrm{~m}$ $[55,59]$. In addition to the effect of medium length scale [60], it has been reported recently that the dispersivity will also increase with NPs size [61]. Therefore, for this reason the dispersion coefficient $(D)$ of NPs at the field scale is assumed to be comparatively larger $\left(\sim 10^{-5} \mathrm{~m}^{2} / \mathrm{s}\right)$ in magnitude than the diffusion coefficient for NPs at the laboratory scale $\left(\sim 10^{-8} \mathrm{~m}^{2} / \mathrm{s}\right.$ for a NP with a diameter of $\left.10 \mathrm{~nm}\right)$, which can be obtained using the Stokes-Einstein equation as follows:

$$
D=\frac{k_{B} T}{3 \pi \mu d_{p}},
$$

where $k_{B}\left[\mathrm{ML}^{2} \mathrm{~T}^{-2} \mathrm{~K}^{-1}\right]$ is the Boltzmann constant, $T[\mathrm{~K}]$ is the absolute temperature, $\mu\left[\mathrm{ML}^{-1} \mathrm{~T}^{-1}\right]$ is the fluid viscosity, and $d_{p}[\mathrm{~L}]$ is the diameter of NPs.

2.4.3. Bulk Density and Porosity. The bulk density and porosity of the porous media can be characterized at any scale and do not vary with either the type of NPs or the operating conditions of the subsurface flow. Idris et al. [62] found that the pore-size distribution, rather than the average pore size, affects the retention of NPs at the microscale. However, no research shows how pore-size distribution affects the retention of NPs at a continuum scale.

2.4.4. Retention Model Constants. The model parameters that typically vary with the size, geometry, and type of NPs (physicochemical coating), mineral types of porous media (quartz, clay, carbonates, etc.), and flow conditions are the parameters associated with a retention model. If we assume that the flow conditions are known and fixed in the subsurface, then there are only two variables, that is, type of NPs and rock composition of porous media that affect the retention parameters. Five unknown parameters $\left(k_{\mathrm{irr}}, k_{\mathrm{ra}}, k_{\mathrm{rd}}\right.$, $\left.s_{1 \max }, s_{2 \max }\right)$ in the retention model are described by (4) and (5). There is no theoretical or empirical basis that could be used to estimate these parameters; therefore, the typical approach to estimate these parameters is by performing a history match of the numerical solution with the effluent concentration data. However, it should be noted that the magnitude of these parameters obtained through history matching the laboratory data cannot be directly used for field scale simulations for two reasons: (i) the history match solution is 
nonunique such that there can be various combinations of these parameters that can match the effluent concentration history and (ii) as discussed earlier, the magnitude of retention constants is a function of flow condition, so the value of retention constants obtained at the laboratory scale would be biased because in most cases the flow velocity at laboratory scale does not mimic the flow velocity in the subsurface. Although history match is a useful tool to obtain retention parameters to validate the model using laboratory data, it does not provide realistic values of these parameters that can be applied at the field scale. For example, the values of retention constants (irreversible and reversible) and the desorption constant in a two-site model obtained by history matching several coreflood effluent concentration data by Zhang [19] are in the range of $10^{-2}-10^{-4} 1 / \mathrm{s}$. However, using these parameter values to perform a field scale simulation would suggest complete retention of NPs in the pore space, which is physically inconsistent with the observations in a pilot-scale injection of NPs [36, 63-67]. As mentioned earlier, there is no theoretical or empirical basis that could be used to estimate these parameters; however, we can use an analogous relationship for the deposition rate constant from colloid filtration theory that has been rigorously tested and applied to model NP retention $[19,39,68,69]$. Our rationale for using an analogous relationship is to obtain a realistic range of retention constant values in a two-site model at disparate scales. This empirical relation, given by (9), is based on mechanisms of deposition, interception, and sedimentation $[36,38,51,70]$ :

$$
k_{\mathrm{dep}}=\frac{3}{2}\left(\frac{1-\phi}{d_{c}}\right) v \alpha \eta_{0},
$$

where $k_{\mathrm{dep}}[1 / \mathrm{T}]$ is the deposition rate constant, $d_{c}[\mathrm{~L}]$ is the diameter of sand grain, $v[\mathrm{~L} / \mathrm{T}]$ is the fluid velocity, $\eta_{0}[-]$ is the single collector removal efficiency, and $\alpha[-]$ is the attachment efficiency representing the ratio of the number of particles adsorbing to the media to the rate at which particles strike the media. The value of $\alpha$ can be determined using the following expression [71, 72]:

$$
\alpha=-\frac{2}{3} \frac{d_{c}}{(1-\phi) \ln } \ln \left(\frac{c}{c_{0}}\right),
$$

where $l[\mathrm{~L}]$ is the length of the media and $c / c_{0}$ is the normalized effluent concentration of NPs at the initial stage of the NP breakthrough curve. Equation (10) can be used to estimate the attachment efficiency of different kinds of NPs to water-saturated porous media under different flow conditions. For most NPs with application in the subsurface, Cullen et al. [39] found $\alpha$ values to be $\sim 2 \times 10^{-4}$.

It can be observed from the above two expressions of $k_{\text {dep }}$ and $\alpha$ that in addition to being a function of rock properties such as porosity, size of media, and diameter of grain, the deposition of NPs is also a function of flow velocity. This means that the retention of NPs at the laboratory scale cannot simply be translated to the field scale without accounting for the change in the deposition constant due to variation in these parameters, especially the flow velocity, because the rock properties do not change significantly from lab to field scale.
The sensitivity of the deposition constant to these variables has been demonstrated by various authors $[19,39,69]$, but the sensitivity with respect to flow velocity is of particular interest here because it can suggest the range of deposition constant values that would exist under subsurface flow conditions. Typical flow velocity in the subsurface is between 0.01 and $1 \mathrm{ft} /$ day, depending on the permeability of the medium. An estimate of the deposition constant obtained at this flow velocity is around $10^{-8} 1 / \mathrm{s}$. This scaling pattern of the retention constant, its tendency to decrease as domain size of the porous media increases, has also been shown mechanistically and verified using stochastic simulations [73-75].

In general, the magnitudes of irreversible retention, reversible retention, and desorption rate constants would lie in the same range $\left(\sim 10^{-8} 1 / \mathrm{s}\right)$ but may vary by a factor of 10 to 60 in some cases, as observed in the parameters obtained by Zhang [19] for a series of experiments conducted by Yu [31] and Murphy [27]. Also, we know that some NPs are always retained at laboratory scale (less than 3\%, [19]), which indicates that the irreversible retention is always bigger than reversible retention or desorption. Therefore, using this observation and the information from laboratory data [19, $27,31]$, we assume rate constants for reversible retention and desorption are about 40 times smaller (on average) than the irreversible retention constant.

Similarly, an approximate range for maximum retention capacity (irreversible and reversible) can be obtained using an analogous relationship from colloid filtration theory [36], which has also been rigorously tested and applied to model NP retention in various studies $[39,69]$

$$
s_{\max }=19.6\left[\left(\frac{v d_{c}}{D}\right)^{1 / 3}\left(\frac{d_{c}}{d_{M}}\right)\right]^{-1.2} .
$$

Retention capacity $\left(s_{\max }\right)$ is inversely proportional to flow velocity $\left(\propto 1 / v^{1.2 / 3}\right)$ and pore size $\left(\propto 1 / d_{c}^{1.2}\right)$; therefore, the values of maximum retention capacities would be lower in laboratory conditions because of the higher flow rates typically used in laboratory experiments. If we assume $S_{1 \max }=5 \times 10^{-6} \mathrm{~g} / \mathrm{g}$ under laboratory conditions [19] with flow velocity of $0.2 \mathrm{ft} / \mathrm{d}$, then $S_{1 \max }$ under reservoir conditions with flow velocity of $0.01 \mathrm{ft} / \mathrm{d}$ would be $\sim(0.2 / 0.01)^{(1.2 / 3)} \times$ $\left(5 \times 10^{-6}\right) \sim 1.66 \times 10^{-5} \mathrm{~g} / \mathrm{g}$. This value lies in the same range as given by Cullen et al. [39] for carbon NPs under subsurface conditions $\left(S_{\max } \sim 1.18 \times 10^{-5} \mathrm{~g} / \mathrm{g}\right)$. However, there could be a variation in magnitudes between irreversible and reversible processes by a factor of 10 to 60 , as shown in the parameters of Zhang [19] obtained by matching a series of coreflood experiments conducted by $\mathrm{Yu}$ [31] and Murphy [27]. On this basis, irreversible retention is usually stronger than reversible retention or desorption, and as explained earlier, we assume that, on average, reversible retention and desorption capacities are about 40 times smaller than the irreversible retention capacity.

\section{Model Parameters at Different Length Scales}

It is hypothesized that the retention of NPs in porous media is a function of porous media length scale, like many other 
TABLE 1: Baseline parameters for six cores at laboratory scale.

\begin{tabular}{lccc}
\hline Parameter & Unit & Value & Remarks \\
\hline$k_{x}$ & $\mathrm{md}$ & 25 & Constant \\
$k_{y}$ & $\mathrm{md}$ & 2.5 & Constant \\
$\phi$ & $\%$ & 10 & Constant \\
$v$ & $\mathrm{ft} / \mathrm{d}$ & 0.2 & Function of porosity and permeability \\
$D$ & $\mathrm{~m}^{2} / \mathrm{s}$ & $10^{-8}$ & Constant; equation (8) \\
$\rho_{b}$ & $\mathrm{~kg} / \mathrm{m}^{3}$ & 2600 & Constant \\
$k_{\text {irr }}$ & $1 / \mathrm{s}$ & $5 \times 10^{-8}$ & Equation (9); Zhang [19] \\
$k_{\text {ra }}$ & $1 / \mathrm{s}$ & $0.125 \times 10^{-8}$ & Equation (9); Zhang [19] \\
$k_{\text {rd }}$ & $1 / \mathrm{s}$ & $0.125 \times 10^{-8}$ & Equation (9); Zhang [19] \\
$s_{1 \max }$ & $\mathrm{g} / \mathrm{g}$ & $5 \times 10^{-6}$ & Equation (11); Zhang [19] \\
$s_{2 \max }$ & $\mathrm{g} / \mathrm{g}$ & $1.25 \times 10^{-7}$ & Equation (11); Zhang [19] \\
$c_{\text {inj }}$ & $\mathrm{kg} / \mathrm{m}^{3}$ & 0.005 & Constant \\
$t_{\text {inj }}$ & $\mathrm{PVs}$ & 4 & Constant
\end{tabular}

physical parameters that are function of scale of investigation $[76,77]$. To test this hypothesis, we need models of porous media at increasing length scales with their appropriate physical $\left(k_{x}, k_{y}, \phi, D\right)$ and chemical $\left(k_{\text {irr }}\right)$ heterogeneity structure. In order to see the effect of length scale on NP retention, we have to use a fixed magnitude for some of the parameters such as subsurface flow velocity to eliminate its bias on NP retention. We investigate the fraction of NPs retained at both small laboratory scale (core) and large field scale (outcrop). At laboratory scale, we model NP retention at six different core lengths, and at field scale we model NP retention at seven different outcrop lengths. Typically, the rock characteristics of laboratory scale core are homogeneous, whereas the rock characteristics at field scale are quite heterogeneous. Therefore, we assume spatially homogeneous parameters in the laboratory scale core models, whereas the field scale models are rendered spatially heterogeneous to mimic the outcrop of a carbonate field.

3.1. Parameters at Laboratory Scale. Figure 2 shows six cores of increasing length having homogeneous properties, which are given in Table 1 . The basis on which some of these parameters were obtained was explained earlier in detail, and a brief remark in this regard as relevant to each parameter is also noted in Table 1.

The six cores at laboratory scale are discretized spatially with the size of one grid cell along horizontal and vertical directions equal to 0.067 inches and 0.15 inches, respectively, for each core.

3.2. Parameters at Field Scale. Field scale models are generated to mimic the spatial heterogeneity of an outcrop from a carbonate field. These seven outcrop sites, shown in Figure 3, come from one single outcrop at increasing distances, as depicted by outcrop images with increasing length in Figure 3. Figure 3 shows seven outcrops of increasing lengths with their corresponding physical $\left(k_{x}, k_{y}, \phi, D\right)$ and chemical $\left(k_{\text {irr }}\right)$ properties defined spatially by six color legends representing six facies, as shown in the inset table in Figure 3.
Each of these five parameters $\left(k_{x}, k_{y}, \phi, D, k_{\text {irr }}\right)$ is extracted for all seven outcrops shown in Figure 3 such that the spatial structure of each parameter mimics the spatial structure of six facies (color legends) in each outcrop image. Using the spatially heterogeneous outcrop images defined by six facies and the corresponding magnitude of each parameter, four parameters representing permeability along $x$-direction, permeability along $y$-direction, porosity, and irreversible retention rate constant, respectively, were rendered spatially heterogeneous in all seven outcrops, as shown in Figures 4-7.

The range of each model parameter at field scale is given in Table 2. The basis for choosing the range of these parameters was explained earlier in detail, and a brief remark in this regard as relevant to each parameter is also noted in Table 2.

The seven outcrops at field scale are discretized spatially with the size of one grid cell along horizontal and vertical directions equal to $10 \mathrm{ft}$ and $5 \mathrm{ft}$, respectively, for each outcrop.

\section{Model Validation with Experimental Data}

To validate the numerical model, we used data from column experiments of NP transport with single-phase flow [27, 31]. In this experiment salt-tolerant silica NPs of $5 \%$ (wt\%) concentration are injected into a water-saturated $1 \mathrm{ft}$ long column sandpack having $48.6 \%$ porosity with a flow velocity of $1038 \mathrm{ft} / \mathrm{d}(=0.012 \mathrm{ft} / \mathrm{s})$. Data from this experiment were used by Zhang [19] to determine the five unknown parameters of a two-site model $\left(k_{\mathrm{irr}}, k_{\mathrm{ra}}, k_{\mathrm{rd}}, s_{1 \mathrm{max}}, s_{2 \max }\right)$ through an optimization program. The estimate of these five retention parameters obtained by Zhang [19] was further verified by using these parameters to simulate and match the effluent concentration data from two more experiments through the same column [27, 31]. The estimate of hydrodynamic dispersion coefficient $D$ used to match their data was not directly reported by the authors.

We used the estimate of five retention parameters $\left(k_{\text {irr }}\right.$, $\left.k_{\text {ra }}, k_{\text {rd }}, s_{1 \text { max }}, s_{2 \max }\right)$ obtained by Zhang [19] and a guessed value of dispersion coefficient $D$ to simulate the NP concentration from our numerical model. The results are then 
TABLE 2: Baseline parameters for seven outcrops at field scale.

\begin{tabular}{lcccc}
\hline Parameter & Unit & Range & Spatially heterogeneous? & Remarks \\
\hline$k_{x}$ & $\mathrm{md}$ & $0.1-50$ & Yes & Outcrop map in Figure 4 \\
$k_{y}$ & $\mathrm{md}$ & $0-5$ & Yes & Outcrop map in Figure 5 \\
$\phi$ & $\%$ & $1-15$ & Yes & Outcrop map in Figure 6 \\
$v$ & $\mathrm{ft} / \mathrm{d}$ & $0.01-1$ & Yes & Function of porosity and permeability \\
$D$ & $\mathrm{~m}^{2} / \mathrm{s}$ & $10^{-5}$ & No & Constant; Liu et al. [59] \\
$\rho_{b}$ & $\mathrm{~kg} / \mathrm{m}^{3}$ & 2600 & No & Constant \\
$k_{\text {irr }}$ & $1 / \mathrm{s}$ & $(2-8) \times 10^{-8}$ & Yes & Equation (9); Zhang [19]; outcrop map in Figure 7 \\
$k_{\text {ra }}$ & $1 / \mathrm{s}$ & $1 \times 10^{-9}$ & No & Equation (9); Zhang [19] \\
$k_{\text {rd }}$ & $1 / \mathrm{s}$ & $1 \times 10^{-4}$ & No & Equation (9); Zhang [19] \\
$s_{1 \max }$ & $\mathrm{g} / \mathrm{g}$ & $1.7 \times 10^{-5}$ & No & Equation (11); Zhang [19] \\
$s_{2 \max }$ & $\mathrm{g} / \mathrm{g}$ & $4.2 \times 10^{-7}$ & No & Equation (11); Zhang [19] \\
$C_{\text {inj }}$ & $\mathrm{wt} \%$ & 0.5 & No & Constant \\
$t_{\text {inj }}$ & $\mathrm{PVs}$ & 4 & & Constant \\
\hline
\end{tabular}

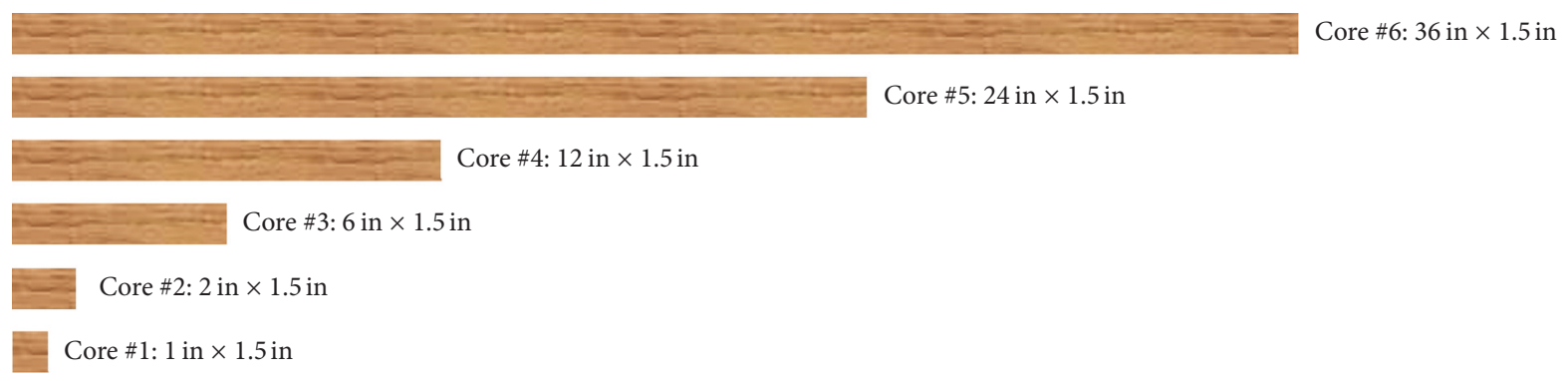

FIGURE 2: Six cores with increasing length at laboratory scale.

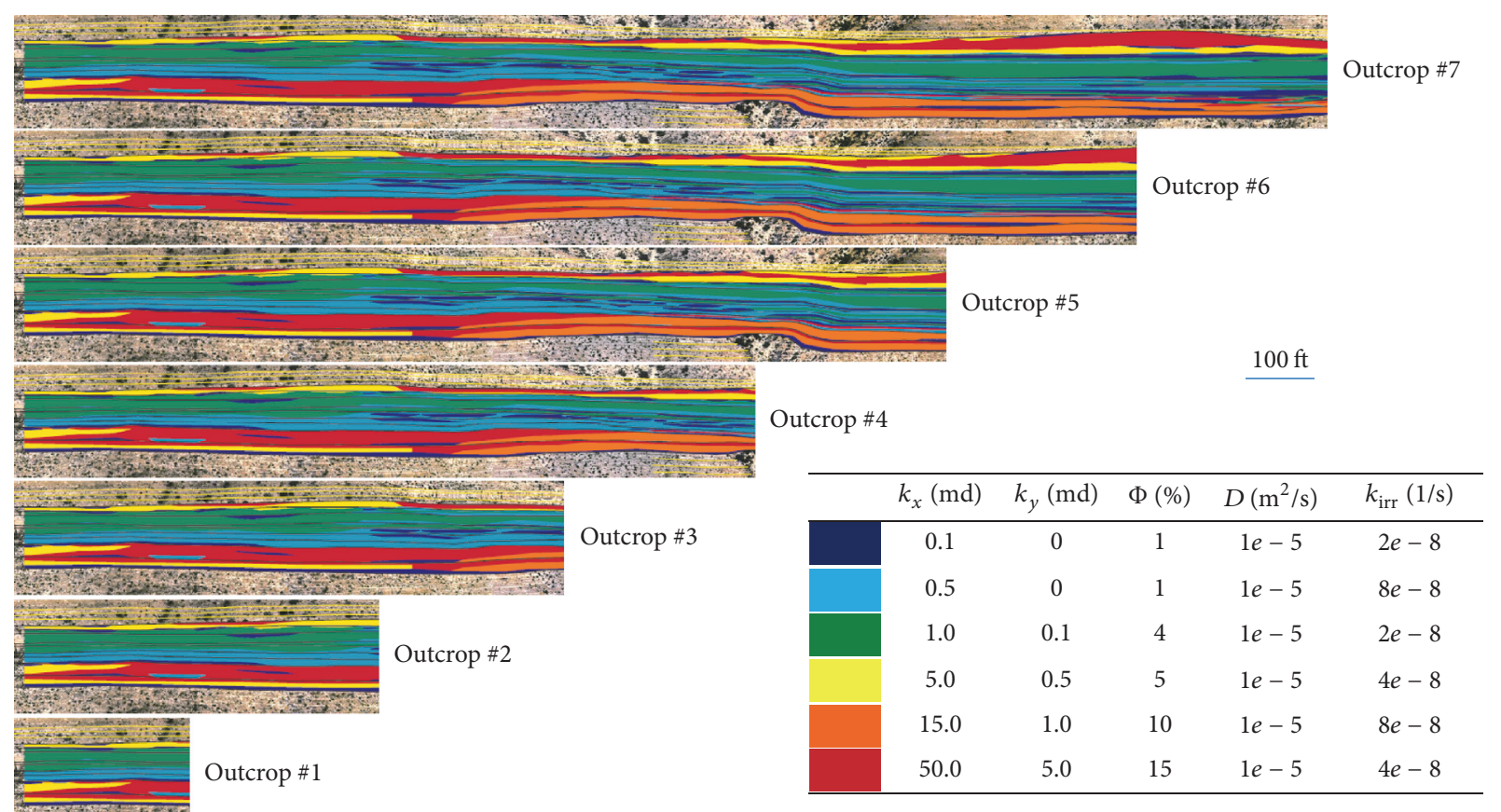

FIGURE 3: Seven images of the same outcrop with its increasing length at field scale. The outcrop comes from a carbonate field having heterogeneous physical and chemical properties $[78,79]$. 

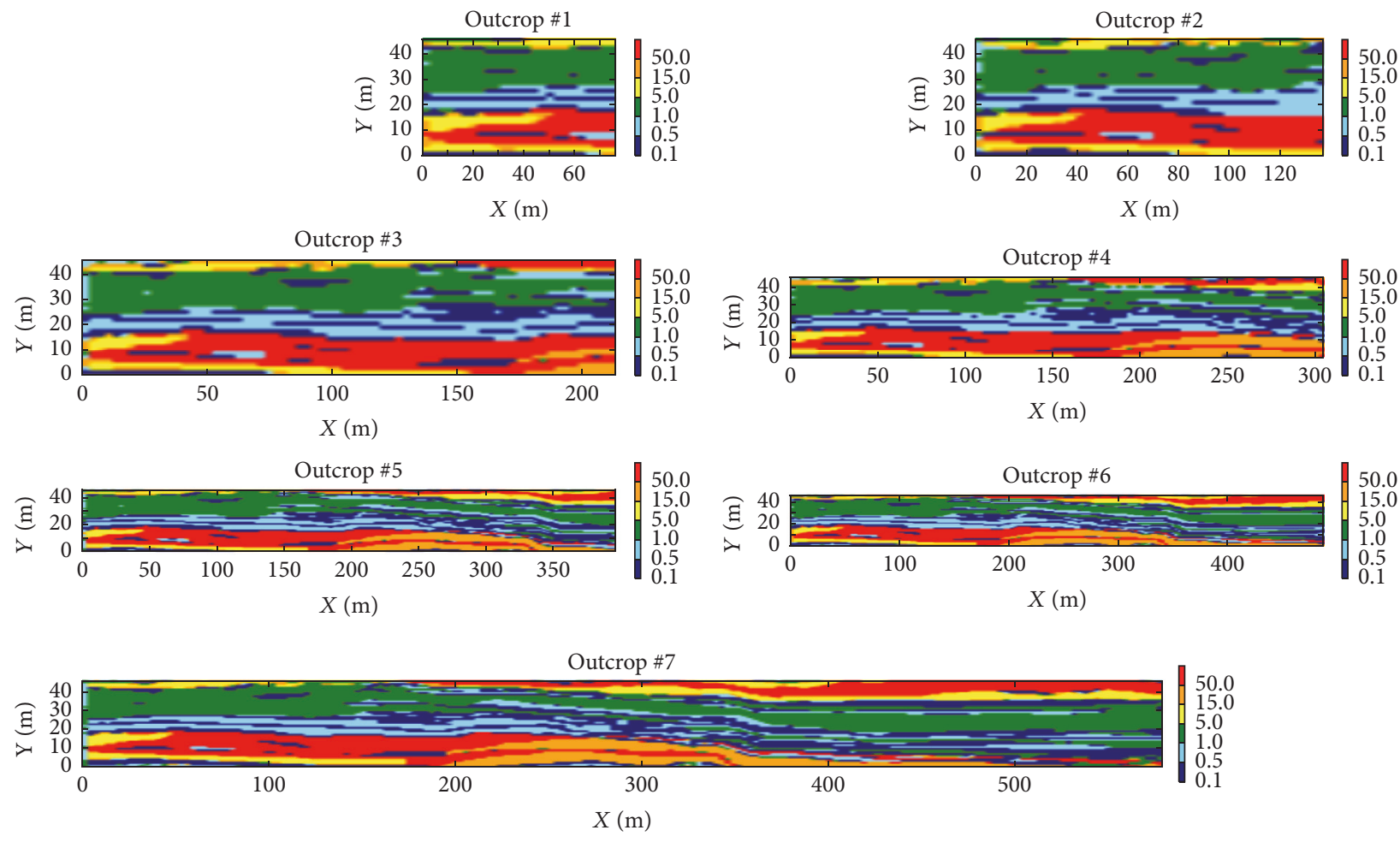

FIGURE 4: Map of $x$-direction permeability (md) for seven outcrops at field scale $[78,79]$.
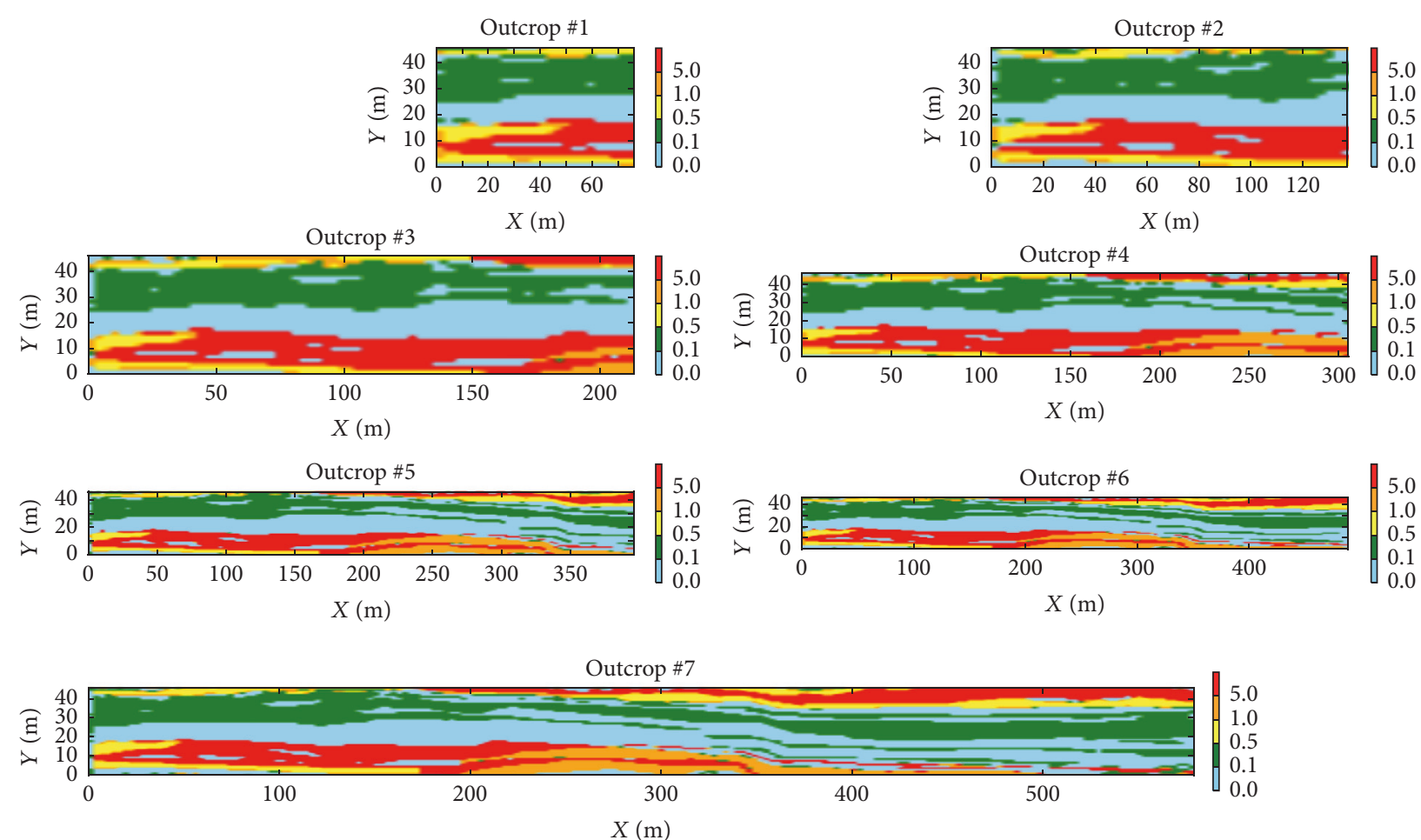

FIGURE 5: Map of $y$-direction permeability (md) for seven outcrops at field [78, 79]. 

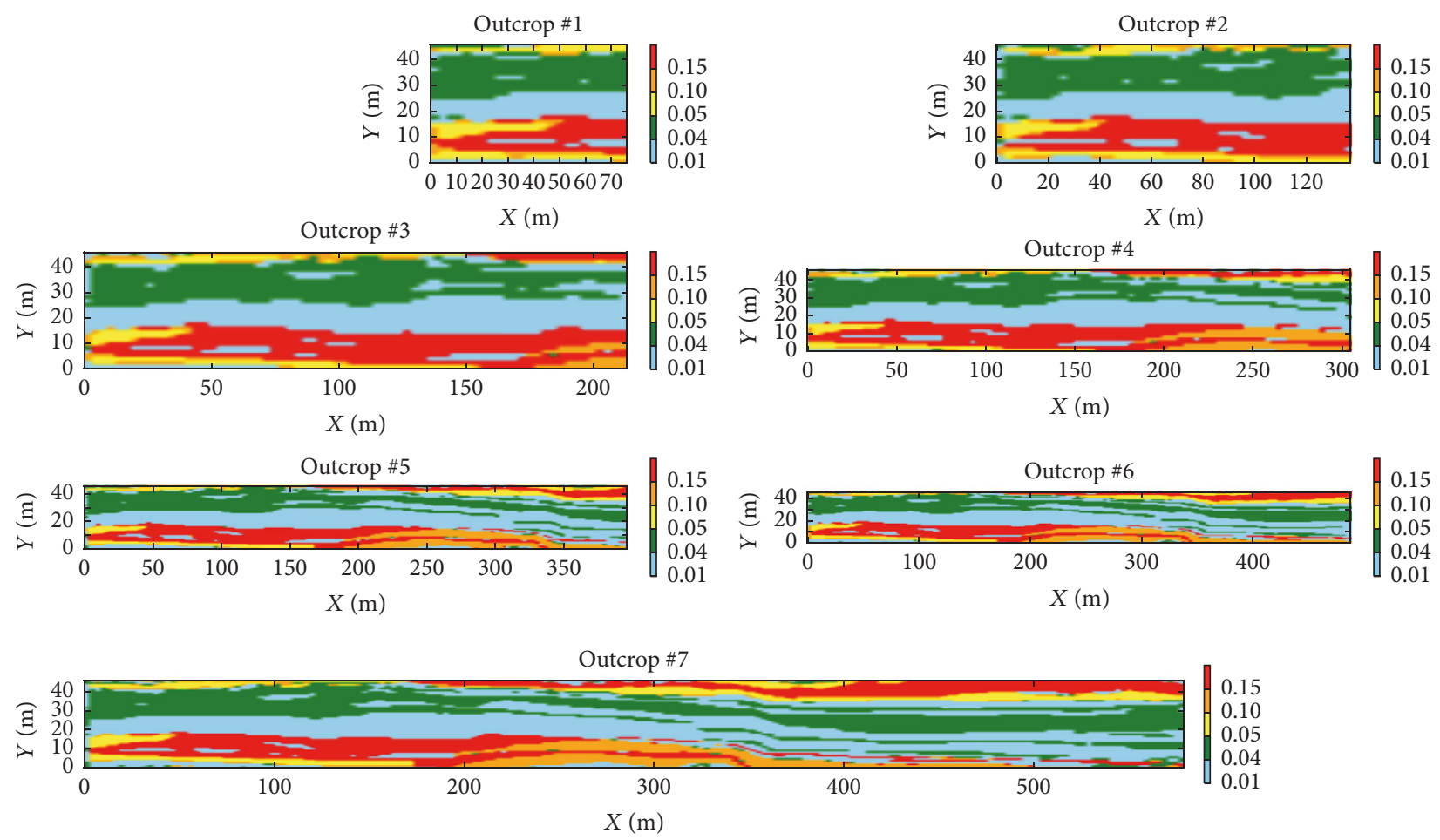

FIgURE 6: Porosity (fraction) map for seven outcrops at field scale [78, 79].

compared with the effluent concentration data of salt-tolerant silica NPs from one of the experiments used by Zhang [19]. The comparison of simulated results for effluent NP concentration from our model and the experimental data is shown in Figure 8. It shows a good match between the experimental data and the simulated curve obtained using the numerical model. The minor difference in the match could be resolved if the available set of optimized parameters included dispersion coefficient.

\section{Results}

The retention of NPs at the laboratory scale and at the field scale is assessed under flow conditions (e.g., velocity and diffusivity) that would typically be present in the field. The physical and chemical characteristics (e.g., porosity, permeability, and retention rate constant) of the porous medium are retained as they would appear at each laboratory and field scale; the physical and chemical properties are assumed homogeneous at laboratory scale, whereas at field scale they are heterogeneous and the structure of their heterogeneity is extracted from outcrop images. The different scales of length assessed, along with the appropriate parameter values for each scale, are presented in Section 3.

Using the history of the effluent concentration and the total concentration of NPs injected, we estimated the fraction of NPs retained inside the porous media for each model at laboratory scale and field scale using the following equation:

$$
\text { Fraction of the NPs adsorbed }=\frac{\left(\mathrm{NPs}_{\mathrm{inj}}-\mathrm{NPs} \mathrm{ejec}_{\mathrm{ec}}\right)}{\mathrm{NPs} \text { inj }},
$$

where $\mathrm{NPs}_{\text {inj }}$ is the total concentration of NPs injected and $\mathrm{NPs}_{\text {ejec }}$ is the total concentration of NPs ejected.

5.1. Retention at Laboratory Scale. To explore the fraction of NPs retained at laboratory scale, transport simulations were conducted for the six cores with increasing lengths shown in Figure 2. The effluent concentration of NPs for the six cores obtained at the end of the simulation run is shown in Figure 9. The values of fluid velocity, hydrodynamic dispersion coefficient, retention rate constants, and retention capacities for each core are shown in the title of each plot. In laboratory scale simulations, the heterogeneity of physical and chemical parameters is neglected. Figure 10 shows the fraction of retained NPs in six cores individually and at distances equal to smaller cores in the longest core. The middle plot in this figure shows that the fraction of retained NPs consistently increases with increasing core length with no NPs retained inside the smallest core while $8 \%$ of NPs are retained inside the longest core. The third plot in Figure 10 shows the fraction of retained NPs at distances equal to those of the smaller cores in the longest core. This plot is used to verify the retention of NPs inside individual cores at different lengths, and it shows that the total fraction of retained NPs increases with travel distance inside the longest core, thus confirming the result of NPs retained inside individual cores of varying lengths. There is a slight difference in results between the two plots in Figure 10, which occurs at smaller distances and disappears with increasing length, indicating that the difference in results at a small distance can be attributed to the effects of boundary conditions. 


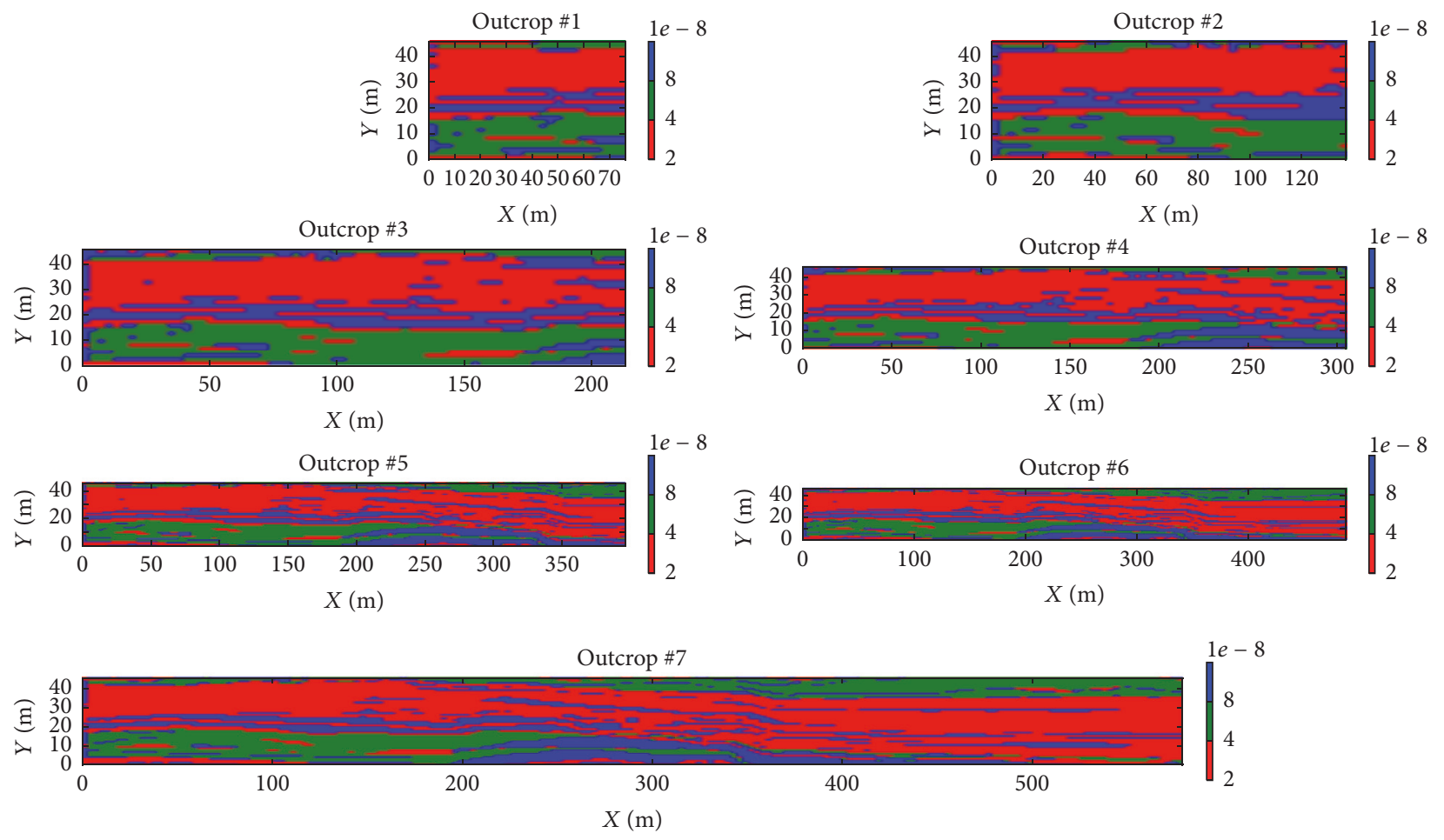

FIGURE 7: Irreversible retention rate constant (1/s) map for seven outcrops at field [78, 79].

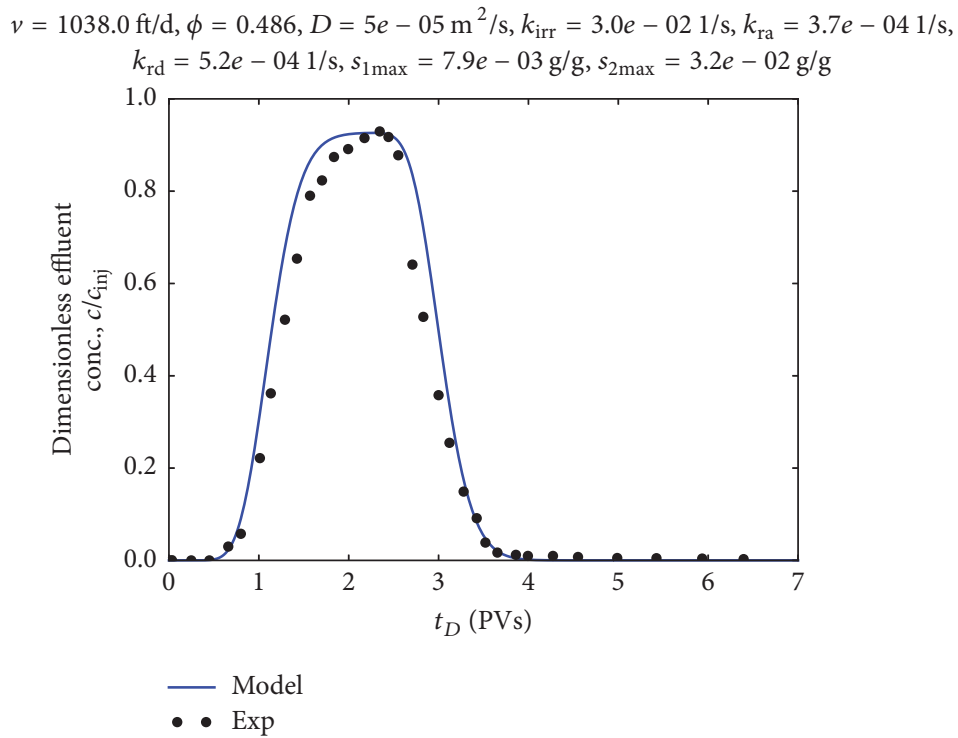

FIGURE 8: Experimental data $[27,31]$ and simulated effluent concentration of NPs. The model parameters used to fit the experimental data come from Zhang [19].

5.2. Retention at Field Scale. To explore the fraction of NPs retained at the field scale, transport simulations were conducted for the seven outcrops with increasing lengths shown in Figure 3. The effluent concentration of NPs for the seven outcrops obtained at the end of the simulation run is shown in Figure 11. The values of fluid velocity, diffusion coefficient, retention rate constants, and retention capacities for each outcrop are shown in the title of each plot. In field scale simulations, the heterogeneity of physical and chemical parameters is represented by outcrop maps shown in Figures $3-7$. Figure 12 shows the fraction of retained NPs in seven outcrops individually and at distances equal to those of the smaller outcrops in the longest outcrop. The middle plot in this figure shows that the fraction of retained NPs consistently increases with increasing outcrop length, with $\sim 92 \%$ of the NPs retained inside the smallest outcrop while $100 \%$ of the 

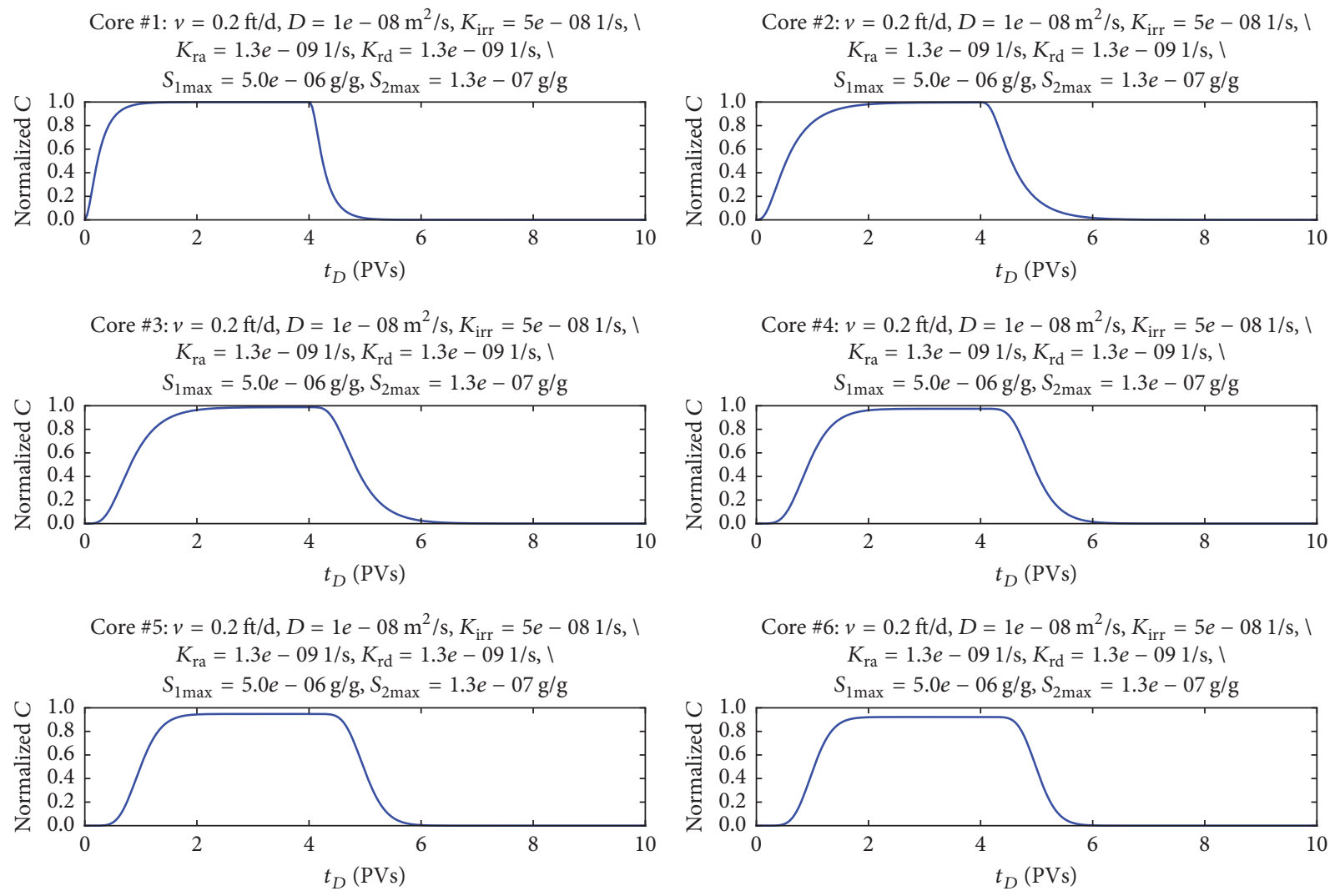

FiguRE 9: Effluent NP concentration for six cores at laboratory scale. The lengths of the six cores considered were 1, 2, 6, 12, 24, and 36 inches, respectively, with 1.5 in width for all the six cores.

NPs are retained inside the five largest outcrops. The third plot in Figure 12 shows the fraction of retained NPs at distances equal to those of the smaller outcrops in the largest outcrop. This plot is used to verify the retention of NPs inside individual outcrops at different lengths, and it shows that the total fraction of retained NPs increases with travel distance inside the largest outcrop, thus confirming the result of NPs retained inside individual outcrops of varying sizes. There is good agreement in results between the two plots in Figure 12, with less than $3 \%$ variation at distances equivalent to smaller outcrops, but this variation disappears with increase in length, indicating that the difference in results at distances equivalent to smaller outcrops can be attributed to the effects of boundary conditions. It should be noted that despite the heterogeneous nature of the outcrop, the result for the fraction of retained NPs in the largest outcrop at distances equal to smaller outcrops is similar to the result inside each individual outcrop because these seven outcrops come from a similar outcrop cut out at increasing distances.

The results of substantially high NP retention at increasing outcrop size support the findings of Cullen et al. [39], who reported almost $50 \%$ retention for nanofullerenes at a scale of $10 \mathrm{~m} \times 8 \mathrm{~m}$ on the basis of field pilot tests. The minimum size of outcrop used in our study is about $75 \mathrm{~m} \times 45 \mathrm{~m}$, and the fraction of NPs retained in an outcrop this size is about $92 \%$.

The slight increase in velocity for outcrop \#7 is due to difficulty in constraining the exact magnitude of velocity due to slight differences in heterogeneity of porosity and permeability fields across the seven outcrop scales. This can be understood mathematically as $v=((k / \phi \mu) \cdot \nabla P)$, where, except for pressure gradient $(\nabla P)$ and fluid viscosity $(\mu)$, other two quantities $(k$ and $\phi)$ are the average of their heterogeneous fields. Consequently, a slight increase $(\sim 0.01 \mathrm{~m} / \mathrm{s})$ of velocity for outcrop \#7 causes an increase in output concentration of NP as visible in Figure 11.

\section{Summary and Conclusion}

The retention of NPs in porous media as a function of length scale was assessed by numerically modeling the injection of NPs in laboratory scale homogeneous cores and field scale heterogeneous outcrops. As discussed earlier, transport and retention of NPs are a function of flow velocity; therefore, to realistically mimic the retention of NPs at the field, a magnitude of flow velocity typically found in the subsurface was chosen and maintained constant across all scales. Additionally, keeping the flow velocity and dispersion coefficient consistent across all scales yields an unbiased comparison when estimating the fraction of retained NPs from small to large scale. The physical and chemical parameters of the porous media were assumed homogeneous at the laboratory scale, whereas four parameters $\left(k_{x}, k_{y}, \phi, k_{\text {irr }}\right)$ were considered spatially heterogeneous at the outcrop scale by matching the spatial structure of six color legends representing six facies of 

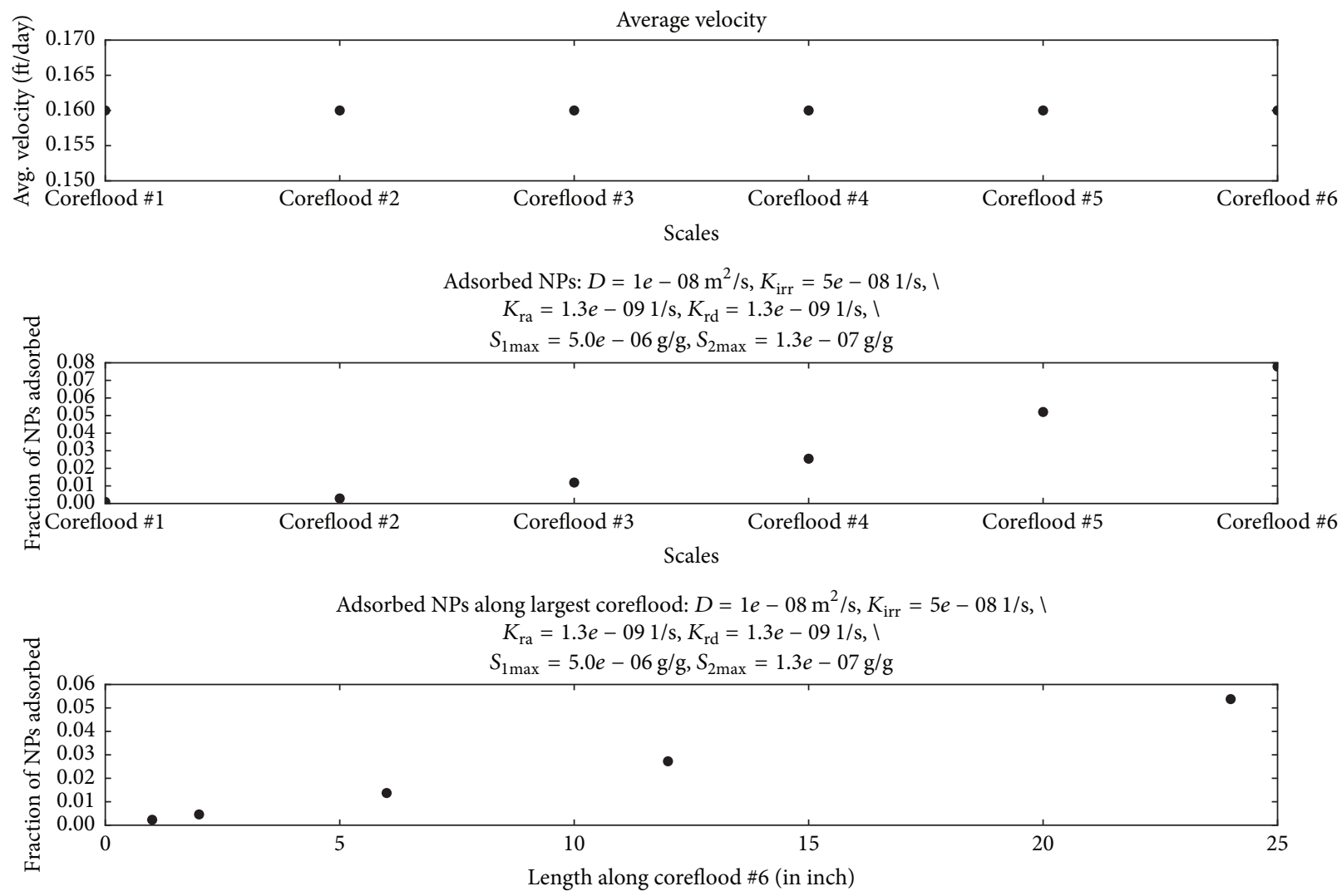

FiguRE 10: Fraction of NPs retained in six cores and at distances equal to smaller cores in the longest core. The results reported are for 4 pore volumes of injected NPs.
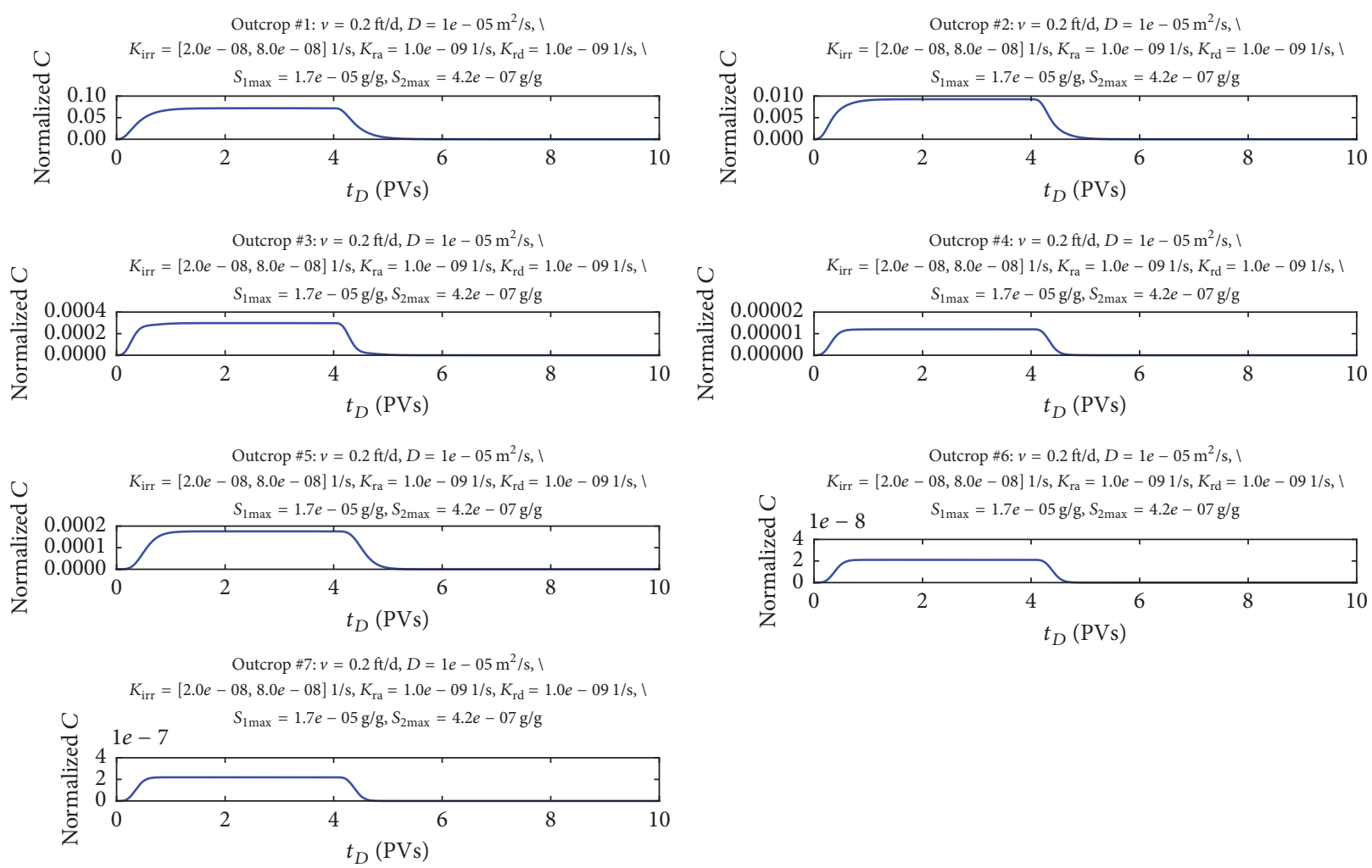

Figure 11: Effluent NP concentration for seven outcrops at field scale. 

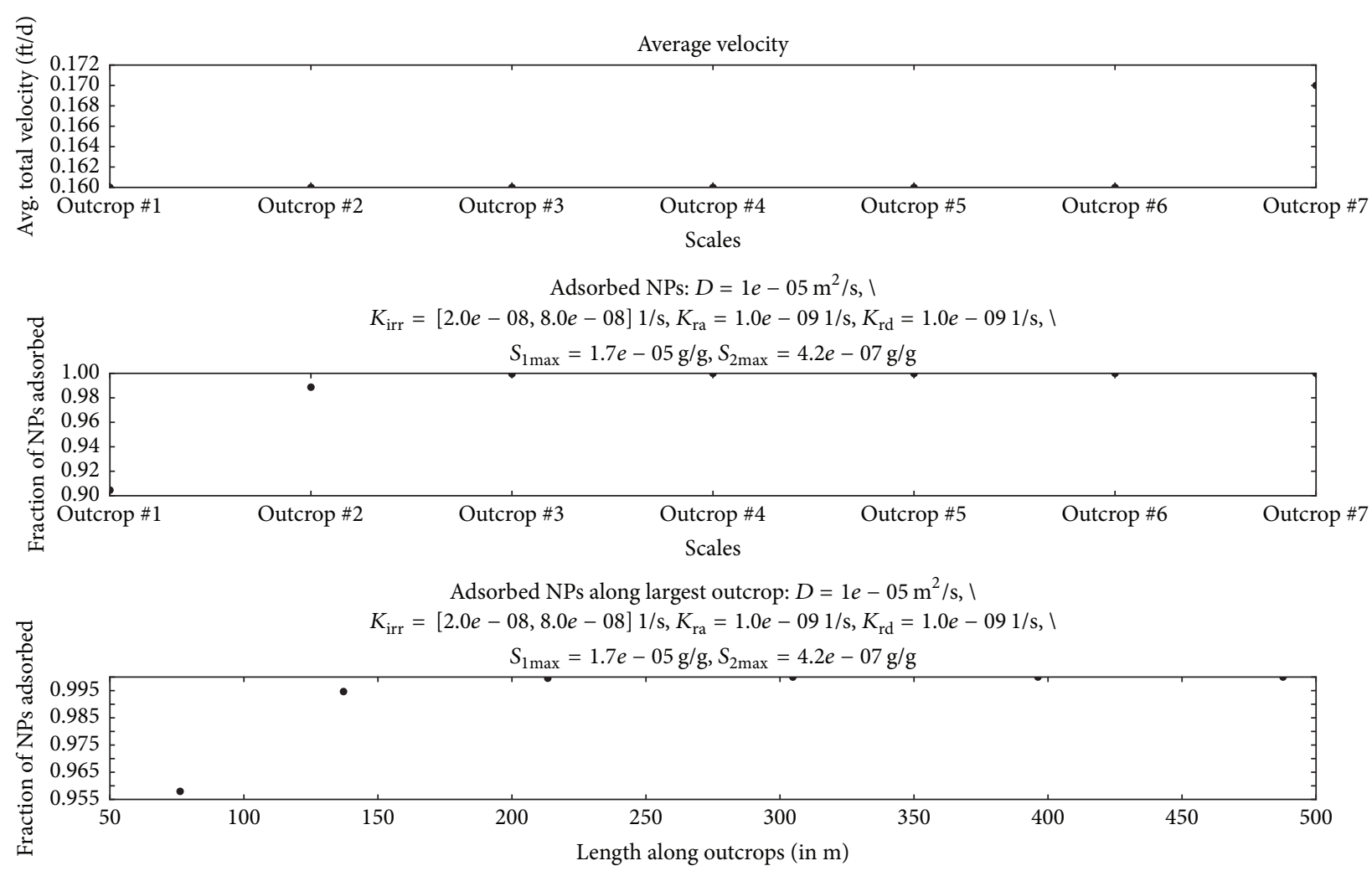

FIGURE 12: Fraction of NPs retained in seven outcrops and at distances equal to smaller outcrops in the longest outcrop. The results reported are for 4 pore volumes of injected NPs.

the carbonate field outcrop (Figure 3). Wettability of different mineral types was included in our simulation by assigning different retention rate constants for different mineral types (different wettability). Results from this study show the following:

(i) NPs can travel hundreds of meter in reservoir at constant velocity.

(ii) The fraction of injected NPs retained in the subsurface increases nonlinearly with the length scale of the homogeneous media and the media with consistent heterogeneity at all scales. There were small changes in heterogeneity at two outcrop scales that affected the fluid flow velocity and that slightly affected the retention behavior of NPs in reference to the behavior expected from homogeneous media or media with consistent heterogeneity.

(iii) The significance of heterogeneity in changing the bulk flow velocity may eventually affect the retention profile of NPs. Therefore, the length of the porous medium and its scale-variant heterogeneity are shown to be the two important parameters that may impact the retention behavior of NPs, which is typically studied at the laboratory scale without much consideration for these two parameters.

\section{Nomenclature}

$k_{x}$ : Permeability along $x$-direction, md

$k_{y}$ : Permeability along $y$-direction, md

$\phi: \quad$ Porosity, \%

v: $\quad$ Total velocity, $\mathrm{ft} / \mathrm{d}$

D: $\quad$ Dispersion coefficient, $\mathrm{m}^{2} / \mathrm{s}$

$\rho_{b}: \quad$ Porous medium bulk density, $\mathrm{kg} / \mathrm{m}^{3}$

$k_{\text {irr: }}:$ Irreversible retention rate constant, $1 / \mathrm{s}$

$k_{\mathrm{ra}}$ : Reversible retention rate constant, $1 / \mathrm{s}$

$k_{\mathrm{rd}}: \quad$ Desorption rate constant, $1 / \mathrm{s}$

$s_{1}$ : Concentration of retained NPs due to irreversible retention, $\mathrm{g} / \mathrm{g}$

$s_{2}$ : Concentration of retained NPs due to reversible retention, $\mathrm{g} / \mathrm{g}$

$s_{1 \max }:$ Capacity for irreversible retention, $\mathrm{g} / \mathrm{g}$

$s_{2 \max }$ : Capacity for reversible retention, $\mathrm{g} / \mathrm{g}$

$c$ : Concentration of NPs within the porous medium, $\mathrm{kg} / \mathrm{m}^{3}$

$c_{\text {inj }}: \quad$ Injected concentration of NPs, $\mathrm{kg} / \mathrm{m}^{3}$

$t_{\text {inj }}: \quad$ Dimensionless time for which NPs are injected, PVs

$k_{B}$ : Boltzmann constant, $\mathrm{J} / \mathrm{K}$

$d_{p}: \quad$ Diameter of NPs, $\mathrm{m}$

$\mu$ : $\quad$ Fluid viscosity, $\mathrm{Pa}-\mathrm{s}$

T: $\quad$ Temperature, $\mathrm{K}$

$k_{\text {dep }}:$ Deposition constant, $1 / \mathrm{s}$ 


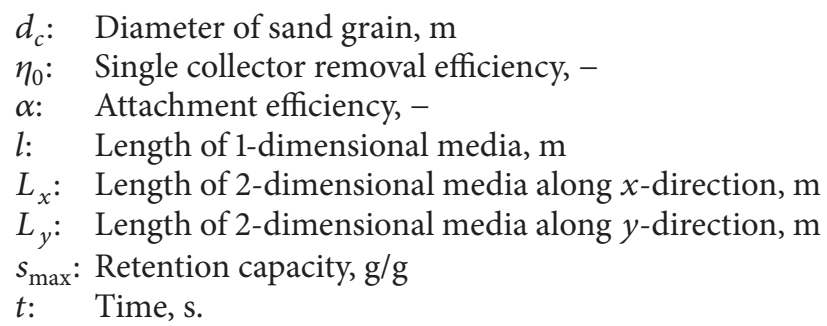

\section{Disclosure}

The current address of Harpreet Singh is National Energy Technology Laboratory, Morgantown, WV, USA.

\section{Conflicts of Interest}

The authors declare that there are no conflicts of interest regarding the publication of this paper.

\section{Acknowledgments}

This work was supported by the NanoGeosciences Laboratory at the University of Texas at Austin. Outcrop images and associated data are provided by the Reservoir Characterization Research Laboratory (RCRL). Susie Doenges edited the manuscript. Publication was authorized by the Director of the Bureau of Economic Geology, the University of Texas at Austin.

\section{References}

[1] S. K. Nune, P. Gunda, P. K. Thallapally, Y.-Y. Lin, M. Laird Forrest, and C. J. Berkland, "Nanoparticles for biomedical imaging," Expert Opinion on Drug Delivery, vol. 6, no. 11, pp. 1175-1194, 2009.

[2] M. V. Yigit and Z. Medarova, " $\mathrm{n}$ vivo and ex vivo applications of gold nanoparticles for biomedical SERS imagingi," American Journal of Nuclear Medicine and Molecular Imaging, vol. 2, pp. 232-241, 2012.

[3] M. Y. Berezin, Nanotechnology for Biomedical Imaging and Diagnostics: From Nanoparticle Design to Clinical Applications, John Wiley \& Sons, 2015.

[4] W. H. de Jong and P. J. A. Borm, "Drug delivery and nanoparticles: applications and hazards," International Journal of Nanomedicine, vol. 3, no. 2, pp. 133-149, 2008.

[5] A. Z. Wilczewska, K. Niemirowicz, K. H. Markiewicz, and H. Car, "Nanoparticles as drug delivery systems," Pharmacological Reports, vol. 64, no. 5, pp. 1020-1037, 2012.

[6] H. Ehtesabi, M. M. Ahadian, V. Taghikhani, and M. H. Ghazanfari, "Enhanced heavy oil recovery in sandstone cores using TiO2 nanofluids," Energy and Fuels, vol. 28, no. 1, pp. 423-430, 2014.

[7] L. Hendraningrat and O. Torsæter, "Metal oxide-based nanoparticles: revealing their potential to enhance oil recovery in different wettability systems," Applied Nanoscience, vol. 5, no. 2, pp. 181-199, 2014.

[8] L. Hendraningrat and O. Torsæter, "A study of water chemistry extends the benefits of using silica-based nanoparticles on enhanced oil recovery," Applied Nanoscience, vol. 6, pp. 83-95, 2015.
[9] K. Xu, P. Zhu, C. Huh, and M. T. Balhoff, "Microfluidic Investigation of Nanoparticles Role in Mobilizing Trapped Oil Droplets in Porous Media," Langmuir, vol. 31, no. 51, pp. 1367313679, 2015.

[10] A. R. Rahmani, M. Ahmadian-Tehrani, and A. E. Athey, "Reservoir characterization and hydraulic fracture evaluation," 2014, https://www.google.com/patents/WO2014144917A1?cl=en.

[11] K. Y. Yoon, The design and control of stability and magnetic properties of imaging nanoparticles [Dissertation], The University of Texas at Austin, 2012.

[12] C. An, Modeling of magnetic nanoparticles transport in shale reservoirs [Ph.D. thesis], 2014.

[13] X. Lv, B. Gao, Y. Sun et al., "Effects of grain size and structural heterogeneity on the transport and retention of nano-TiO2 in saturated porous media," Science of the Total Environment, vol. 563-564, pp. 987-995, 2016.

[14] J. Cai, X. Hu, B. Xiao, Y. Zhou, and W. Wei, "Recent developments on fractal-based approaches to nanofluids and nanoparticle aggregation," International Journal of Heat and Mass Transfer, vol. 105, pp. 623-637, 2017.

[15] W. Wei, J. Cai, X. Hu, Q. Han, S. Liu, and Y. Zhou, "Fractal anal$y$ sis of the effect of particle aggregation distribution on thermal conductivity of nanofluids," Physics Letters A, vol. 380, no. 37, pp. 2953-2956, 2016.

[16] F. M. Caldelas, M. Murphy, C. Huh, and S. L. Bryant, Factors Governing Distance of Nanoparticle Propagation in Porous Media, Society of Petroleum Engineers, 2011.

[17] A. Esfandyari Bayat, R. Junin, S. Shamshirband, and W. Tong Chong, "Transport and retention of engineered Al2O3, TiO2, and $\mathrm{SiO} 2$ nanoparticles through various sedimentary rocks," Scientific Reports, vol. 5, article 14264, 2015.

[18] A. T. Kaasa, Investigation of how Silica Nanoparticle Adsorption Affects Wettability in Water-Wet Berea Sandstone, Norwegian University of Science and Technology, 2013.

[19] T. Zhang, Modeling of nanoparticle transport in porous media [Dissertation], The University of Texas at Austin, 2012.

[20] M. Ranka, P. Brown, and T. A. Hatton, "Responsive stabilization of nanoparticles for extreme salinity and high-temperature reservoir applications," ACS Applied Materials and Interfaces, vol. 7, no. 35, pp. 19651-19658, 2015.

[21] E. Rodriguez Pin, M. Roberts, H. Yu, C. Huh, and S. L. Bryant, Enhanced Migration of Surface-Treated Nanoparticles in Sedimentary Rocks, Society of Petroleum Engineers.

[22] H. Yu, Y. He, P. Li et al., "Flow enhancement of waterbased nanoparticle dispersion through microscale sedimentary rocks," Scientific Reports, vol. 5, article 8702, 2015.

[23] N. S. Lenchenkov, M. Slob, E. Van Dalen, G. Glasbergen, and C. Van Kruijsdijk, Oil Recovery from Outcrop Cores with Polymeric Nano-Spheres, Society of Petroleum Engineers, 2016.

[24] W. Chen, L. Duan, and D. Zhu, "Adsorption of polar and nonpolar organic chemicals to carbon nanotubes," Environmental Science and Technology, vol. 41, no. 24, pp. 8295-8300, 2007.

[25] X. Ma, D. Anand, X. Zhang, M. Tsige, and S. Talapatra, "Carbon nanotube-textured sand for controlling bioavailability of contaminated sediments," Nano Research, vol. 3, no. 6, pp. 412-422, 2010.

[26] X. Ma, D. Anand, X. Zhang, and S. Talapatra, "Adsorption and desorption of chlorinated compounds from pristine and thermally treated multiwalled carbon nanotubes," Journal of Physical Chemistry C, vol. 115, no. 11, pp. 4552-4557, 2011. 
[27] M. J. Murphy, Experimental analysis of electrostatic and hydrodynamic forces affecting nanoparticle retention in porous media [Ph.D. thesis], The University of Texas at Austin, 2012.

[28] B. Pan, D. Lin, H. Mashayekhi, and B. Xing, "Adsorption and hysteresis of bisphenol A and $17 \alpha$-ethinyl estradiol on carbon nanomaterials," Environmental Science and Technology, vol. 42, no. 15, pp. 5480-5485, 2008.

[29] K. S. Sorbie, Polymer-Improved Oil Recovery, 1991.

[30] K. Yang, L. Zhu, and B. Xing, "Adsorption of polycyclic aromatic hydrocarbons by carbon nanomaterials," Environmental Science and Technology, vol. 40, no. 6, pp. 1855-1861, 2006.

[31] H. Yu, Transport and retention of surface-modified nanoparticles in sedimentary rocks [Dissertation], The University of Texas at Austin, 2012.

[32] J. Li and S. Ghoshal, "Comparison of the transport of the aggregates of nanoscale zerovalent iron under vertical and horizontal flow," Chemosphere, vol. 144, pp. 1398-1407, 2016.

[33] D. P. Jaisi, N. B. Saleh, R. E. Blake, and M. Elimelech, "Transport of single-walled carbon nanotubes in porous media: filtration mechanisms and reversibility," Environmental Science and Technology, vol. 42, no. 22, pp. 8317-8323, 2008.

[34] C.-H. Ko and M. Elimelech, “The 'shadow effect' in colloid transport and deposition dynamics in granular porous media: Measurements and mechanisms," Environmental Science and Technology, vol. 34, no. 17, pp. 3681-3689, 2000.

[35] C.-H. Ko, S. Bhattacharjee, and M. Elimelech, "Coupled influence of colloidal and hydrodynamic interactions on the RSA dynamic blocking function for particle deposition onto packed spherical collectors," Journal of Colloid and Interface Science, vol. 229, no. 2, pp. 554-567, 2000.

[36] Y. Li, Y. Wang, K. D. Pennell, and L. M. A. Briola, "Investigation of the transport and deposition of fullerene (C60) nanoparticles in quartz sands under varying flow conditions," Environmental Science and Technology, vol. 42, no. 19, pp. 7174-7180, 2008.

[37] N. B. Saleh, L. D. Pfefferle, and M. Elimelech, "Aggregation kinetics of multiwalled carbon nanotubes in aquatic systems: measurements and environmental implications," Environmental Science and Technology, vol. 42, no. 21, pp. 7963-7969, 2008.

[38] N. Tufenkji and M. Elimelech, "Correlation equation for predicting single-collector efficiency in physicochemical filtration in saturated porous media," Environmental Science and Technology, vol. 38, no. 2, pp. 529-536, 2004.

[39] E. Cullen, D. M. O'Carroll, E. K. Yanful, and B. Sleep, "Simulation of the subsurface mobility of carbon nanoparticles at the field scale," Advances in Water Resources, vol. 33, no. 4, pp. 361371, 2010.

[40] M. F. El-Amin, J. Kou, S. Sun, and A. Salama, "Numerical modeling of nanoparticles transport with two-phase flow in porous media using iterative implicit method," https://arxiv.org/ abs/1310.4769.

[41] M. D. Becker, Y. Wang, K. D. Pennell, and L. M. Abriola, "A multi-constituent site blocking model for nanoparticle and stabilizing agent transport in porous media," Environmental Science: Nano, vol. 2, no. 2, pp. 155-166, 2015.

[42] N. Sun, M. Elimelech, N.-Z. Sun, and J. N. Ryan, "A novel twodimensional model for colloid transport in physically and geochemically heterogeneous porous media," Journal of Contaminant Hydrology, vol. 49, no. 3-4, pp. 173-199, 2001.

[43] H. Singh, S. A. Hosseini, and F. Javadpour, Enhanced CO2 Storage In Deep Saline Aquifers By Nanoparticles: Numerical Simulation Results, Society of Petroleum Engineers, 2012.
[44] P. Babakhani, F. Fagerlund, A. Shamsai, G. V. Lowry, and T. Phenrat, "Modified MODFLOW-based model for simulating the agglomeration and transport of polymer-modified $\mathrm{Fe}^{0}$ nanoparticles in saturated porous media," Environmental Science and Pollution Research, pp. 1-20, 2015.

[45] T. Zhang, M. J. Murphy, H. Yu et al., "Investigation of nanoparticle adsorption during transport in porous media," SPE Journal, vol. 20, no. 4, pp. 667-677, 2015.

[46] T. Zhang, M. Murphy, H. Yu, C. Huh, and S. L. Bryant, "Mechanistic model for nanoparticle retention in porous media," Transport in Porous Media, pp. 1-20, 2016.

[47] H. Singh and P. N. Azom, Integration of Nonempirical Shale Permeability Model in a Dual-Continuum Reservoir Simulator, Society of Petroleum Engineers, 2013.

[48] H. Singh and F. Javadpour, A New Non-Empirical Approach to Model Transport of Fluids in Shale Gas Reservoirs, Society of Exploration Geophysicists, American Association of Petroleum Geologists, Society of Petroleum Engineers, 2013.

[49] E. Goldberg, M. Scheringer, T. D. Bucheli, and K. Hungerbühler, "Critical assessment of models for transport of engineered nanoparticles in saturated porous media," Environmental Science and Technology, vol. 48, no. 21, pp. 12732-12741, 2014.

[50] Y. Wang, Y. Li, J. D. Fortner, J. B. Hughes, L. M. Abriola, and K. D. Pennell, "Transport and retention of nanoscale C60 aggregates in water-saturated porous media," Environmental Science and Technology, vol. 42, no. 10, pp. 3588-3594, 2008.

[51] L. Xueying, D. M. O’Carroll, E. J. Petersen, H. Qingguo, and C. L. Anderson, "Mobility of multiwalled carbon nanotubes in porous media," Environmental Science and Technology, vol. 43, no. 21, pp. 8153-8158, 2009.

[52] M. W. Becker and A. M. Shapiro, "Tracer transport in fractured crystalline rock: Evidence of nondiffusive breakthrough tailing," Water Resources Research, vol. 36, no. 7, pp. 1677-1686, 2000.

[53] Liu, Zhang, and Molz, Scale Dependence of the Effective Matrix Diffusion Coefficient: Evidence and Preliminary Interpretation, Lawrence Berkeley National Laboratory, Berkeley, CA, USA, 2006.

[54] H.-H. Liu, C. B. Haukwa, C. F. Ahlers, G. S. Bodvarsson, A. L. Flint, and W. B. Guertal, "Modeling flow and transport in unsaturated fractured rock: an evaluation of the continuum approach," Journal of Contaminant Hydrology, vol. 62-63, pp. 173-188, 2003.

[55] H. H. Liu, G. S. Bodvarsson, and G. Zhang, "Scale dependency of the effective matrix diffusion coefficient," Vadose Zone Journal, vol. 3, no. 1, pp. 312-315, 2004.

[56] I. Neretnieks, "A stochastic multi-channel model for solute transport-analysis of tracer tests in fractured rock," Journal of Contaminant Hydrology, vol. 55, no. 3-4, pp. 175-211, 2002.

[57] A. M. Shapiro, "Effective matrix diffusion in kilometerscale transport in fractured crystalline rock," Water Resources Research, vol. 37, no. 3, pp. 507-522, 2001.

[58] Q. Zhou, H.-H. Liu, F. J. Molz, Y. Zhang, and G. S. Bodvarsson, "Field-scale effective matrix diffusion coefficient for fractured rock: results from literature survey," Journal of Contaminant Hydrology, vol. 93, pp. 161-187, 2007.

[59] H. H. Liu, Y. Q. Zhang, Q. Zhou, and F. J. Molz, "An interpretation of potential scale dependence of the effective matrix diffusion coefficient," Journal of Contaminant Hydrology, vol. 90, no. 1-2, pp. 41-57, 2007.

[60] L. W. Gelhar and C. L. Axness, "Three-dimensional stochastic analysis of macrodispersion in aquifers," Water Resources Research, vol. 19, no. 1, pp. 161-180, 1983. 
[61] C. V. Chrysikopoulos and V. E. Katzourakis, "Colloid particle size-dependent dispersivity," Water Resources Research, vol. 51, no. 6, pp. 4668-4683, 2015.

[62] S. A. Idris, K. M. Alotaibi, T. A. Peshkur, P. Anderson, M. Morris, and L. T. Gibson, "Adsorption kinetic study: effect of adsorbent pore size distribution on the rate of $\mathrm{Cr}$ (VI) uptake," Microporous and Mesoporous Materials, vol. 165, pp. 99-105, 2013.

[63] L. M. Abriola, C. D. Drummond, E. J. Hahn et al., "Pilot-scale demonstration of surfactant-enhanced PCE solubilization at the Bachman Road site. 1. Site characterization and test design," Environmental Science and Technology, vol. 39, no. 6, pp. 17781790, 2005.

[64] J. A. Christ, L. D. Lemke, and L. M. Abriola, "Comparison of two-dimensional and three-dimensional simulations of dense nonaqueous phase liquids (DNAPLs): migration and entrapment in a nonuniform permeability field," Water Resources Research, vol. 41, no. 1, pp. 1-12, 2005.

[65] L. D. Lemke, L. M. Abriola, and J. R. Lang, "Influence of hydraulic property correlation on predicted dense nonaqueous phase liquid source zone architecture, mass recovery and contaminant flux," Water Resources Research, vol. 40, no. 12, artcile W12417, pp. 1-18, 2004.

[66] K. Pulskamp, S. Diabaté, and H. F. Krug, "Carbon nanotubes show no sign of acute toxicity but induce intracellular reactive oxygen species in dependence on contaminants," Toxicology Letters, vol. 168, no. 1, pp. 58-74, 2007.

[67] C. A. Ramsburg, L. M. Abriola, K. D. Pennell et al., "Stimulated microbial reductive dechlorination following surfactant treatment at the Bachman Road site," Environmental Science and Technology, vol. 38, no. 22, pp. 5902-5914, 2004.

[68] T. Rahman, H. Millwater, and H. J. Shipley, "Modeling and sensitivity analysis on the transport of aluminum oxide nanoparticles in saturated sand: effects of ionic strength, flow rate, and nanoparticle concentration," Science of the Total Environment, vol. 499, no. 1, pp. 402-412, 2014.

[69] D. Su, R. Ma, M. Salloum, and L. Zhu, "Multi-scale study of nanoparticle transport and deposition in tissues during an injection process," Medical \& Biological Engineering \& Computing, vol. 48, no. 9, pp. 853-863, 2010.

[70] C. Tien and B. V. Ramarao, Granular Filtration of Aerosols and Hydrosols, Elsevier, 2011.

[71] M. Elimelech, "Effect of particle size on the kinetics of particle deposition under attractive double layer interactions," Journal of Colloid and Interface Science, vol. 164, no. 1, pp. 190-199, 1994.

[72] K.-M. Yao, M. T. Habibian, and C. R. O’Melia, "Water and waste water filtration: concepts and applications," Environmental Science and Technology, vol. 5, no. 11, pp. 1105-1112, 1971.

[73] Z. Dai, A. Wolfsberg, Z. Lu, and H. Deng, "Scale dependence of sorption coefficients for contaminant transport in saturated fractured rock," Geophysical Research Letters, vol. 36, no. 1, 2009.

[74] H. Deng, Upscaling reactive transport parameters for porous and fractured porous media [Dissertation], Florida State University, 2009.

[75] H. Deng, Z. Dai, A. Wolfsberg, Z. Lu, M. Ye, and P. Reimus, "Upscaling of reactive mass transport in fractured rocks with multimodal reactive mineral facies," Water Resources Research, vol. 46 , no. $6,2010$.

[76] H. Singh, Scale-up of reactive processes in heterogeneous media [Dissertation], The University of Texas at Austin, 2014.

[77] H. Singh, "Representative elementary volume (REV) in spatiotemporal domain: a method to find REV for dynamic pores," Journal of Earth Science, vol. 28, no. 2, pp. 391-403, 2017.
[78] C. Kerans and K. Kempter, Hierarchical Stratigraphic Analysis of a Carbonate Platform, Permian of the Guadalupe Mountains, University of Texas, Austin, Bureau of Economic Geology, 2002.

[79] C. Kerans, F. J. Lucia, and R. K. Senger, "Integrated characterization of carbonate ramp reservoirs using Permian San Andres Formation outcrop analogs," Bulletin - American Association of Petroleum Geologists, vol. 78, pp. 181-216, 1994. 

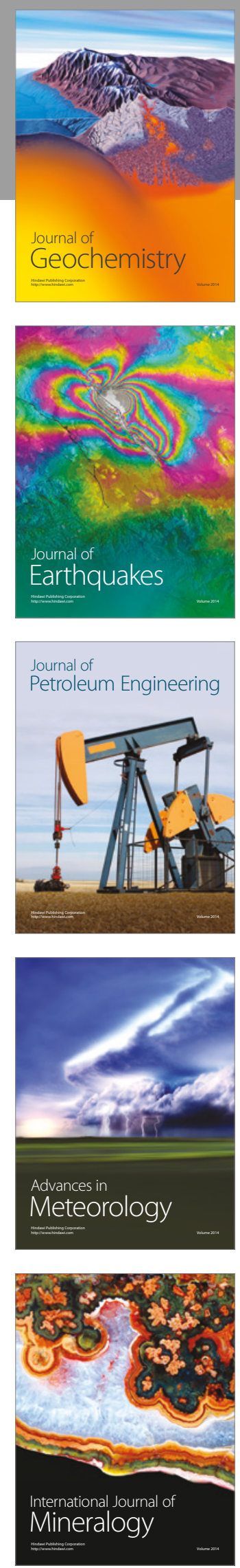
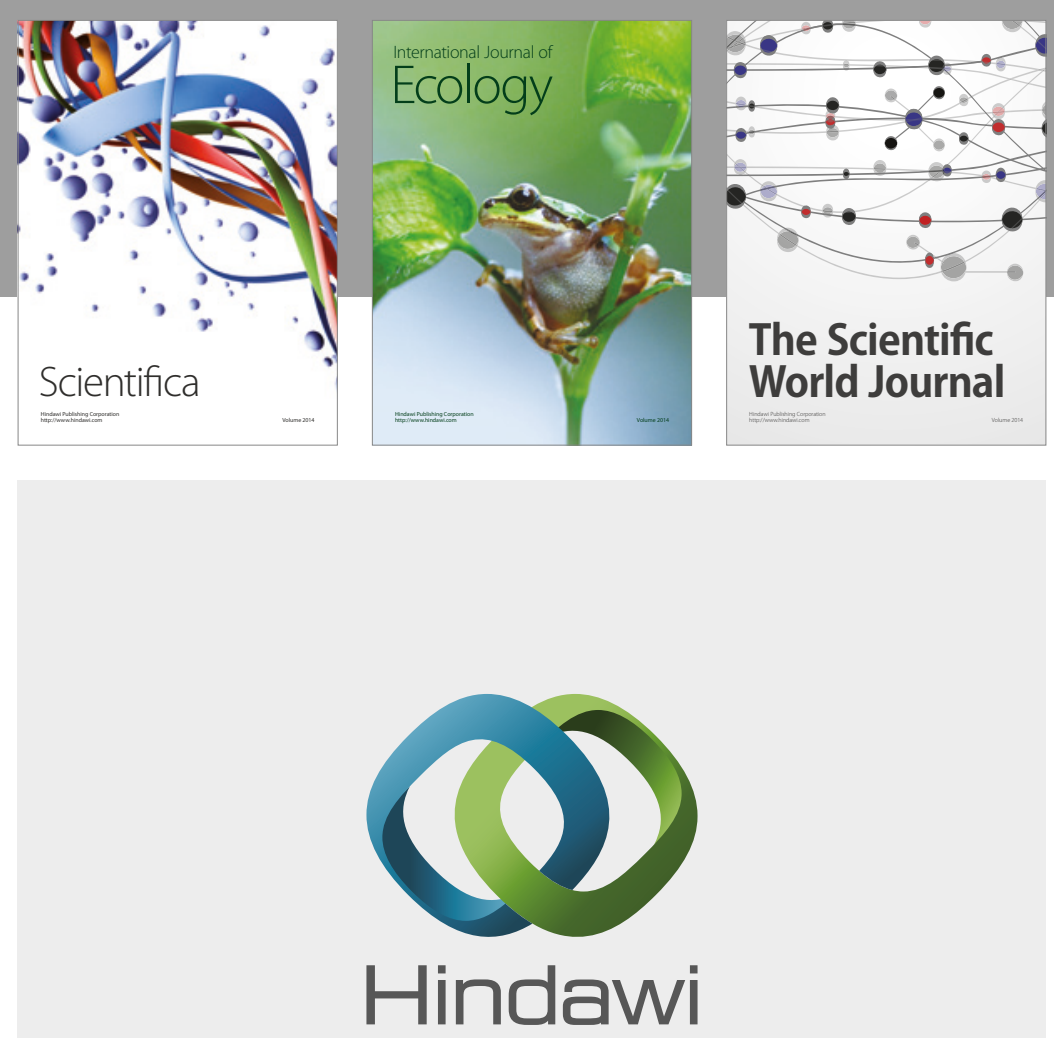

Submit your manuscripts at

https://www.hindawi.com
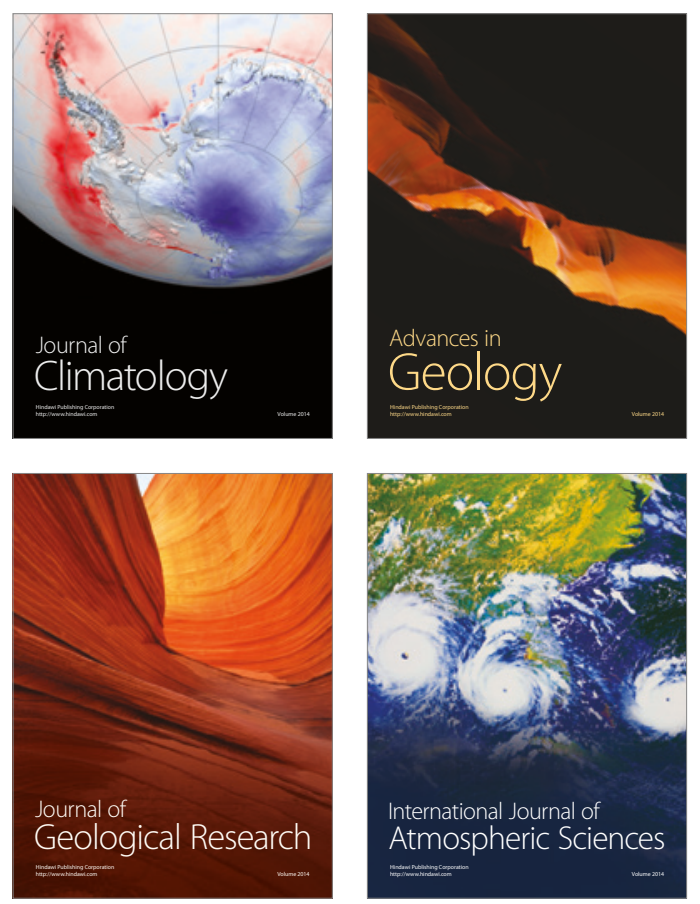

The Scientific

World Journal
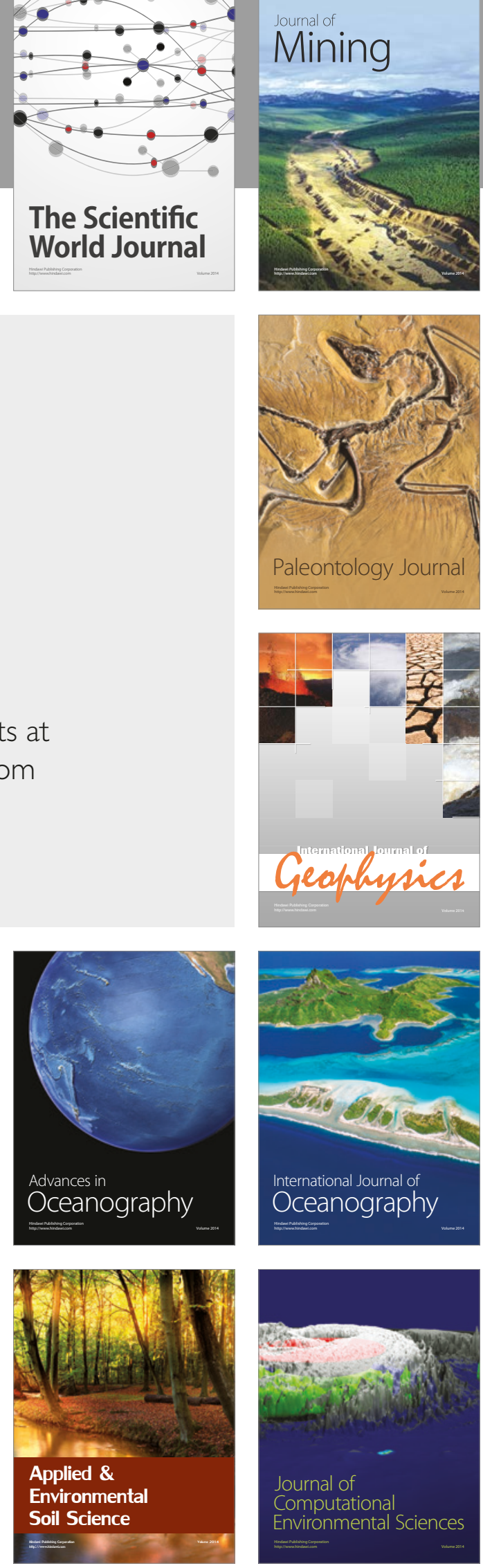